\title{
DEVELOPMENT OF A PERCEPTUAL HYPERTHERMIA INDEX TO EVALUATE HEAT STRAIN DURING TREADMILL EXERCISE
}

\author{
by \\ Michael Gallagher, Jr. \\ B.S.E., University of Pittsburgh, 2003 \\ M.S., University of Pittsburgh, 2007 \\ Submitted to the Graduate Faculty of \\ School of Education in partial fulfillment \\ of the requirements for the degree of \\ Doctorate in Philosophy in Exercise Physiology
}

University of Pittsburgh 


\section{UNIVERSITY OF PITTSBURGH \\ SCHOOL OF EDUCATION}

This dissertation was presented

by

Michael Gallagher, Jr.

It was presented for defense on

December 3, 2009

and approved by

Elizabeth F. Nagle, Ph.D., Assistant Professor

Health and Physical Activity, University of Pittsburgh

David Hostler, Ph.D., Assistant Professor

Emergency Medicine, University of Pittsburgh

Fredric L. Goss, Ph.D., Associate Professor Health and Physical Activity, University of Pittsburgh

Mark A. Schafer, Ph.D., Assistant Professor

Kinesiology, Recreation, and Sport, Western Kentucky University

Dissertation Advisor: Robert J. Robertson, Ph.D., Professor

Health and Physical Activity, University of Pittsburgh 


\title{
DEVELOPMENT OF A PERCEPTUAL HYPERTHERMIA INDEX TO EVALUATE HEAT STRAIN DURING TREADMILL EXERCISE
}

\author{
Michael Gallagher, Jr., Ph.D. \\ University of Pittsburgh, 2009
}

Fire suppression and rescue is a physiologically demanding occupation due to extreme external heat as well as the additional physical and thermal burden of the protective garments. The hot environment challenges body temperature homeostasis inducing heat stress. Accurate field assessment of hyperthermia is complex and unreliable. Purpose: The present investigation developed a perceptually based hyperthermia metric to measure physiologic exertional heat strain during treadmill exercise. Methods: Sixty-five $(28.88 \pm 6.75 \mathrm{yrs})$ female $(\mathrm{n}=11)$ and male $(\mathrm{n}=54)$ firefighters and non-firefighting volunteers participated in four related thermal stress investigations performing treadmill exercise while wearing thermal protective clothing in a heated room. Physiological and perceptual responses (i.e. body core temperature, perceived exertion, and thermal sensation) were assessed at baseline, 20-mins exercise, and at termination. Results: Perceived exertion increased from baseline $(0.24 \pm 0.42)$ to termination $(7.43 \pm$ 1.86). Thermal sensation increased from baseline $(1.78 \pm 0.77)$ to termination $(4.50 \pm 0.68)$. Perceived exertion and thermal sensation were measured concurrently with body core temperature to develop a twodimensional graphical representation of three "colored" exertional heat strain zones. Each exertional heat strain zone was representative of a range of mean body core temperature responses such that green incorporated 36.0 to $37.4^{\circ} \mathrm{C}$, yellow incorporated 37.5 to $37.9^{\circ} \mathrm{C}$, and red incorporated 38.0 to greater than $40.5^{\circ} \mathrm{C}$. Conclusions: A perceptual hyperthermia index (PHI) was developed using ratings of 
perceived exertion and thermal sensation. The PHI can provide a quick and easy momentary assessment of the level of risk for exertional heat strain for firefighters engaged in fire suppression and rescue. This metric may be beneficial in high risk environments that threaten the lives of firefighters. 
TABLE OF CONTENTS

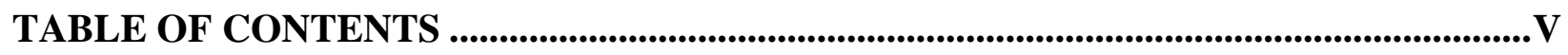

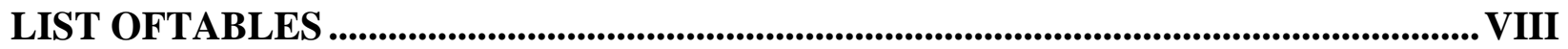

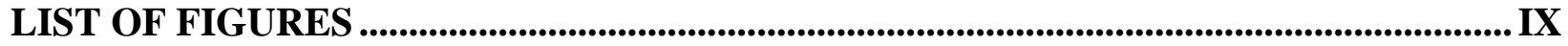

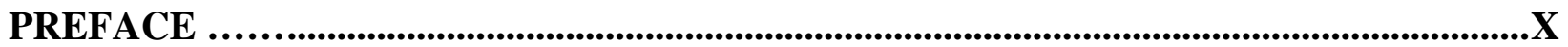

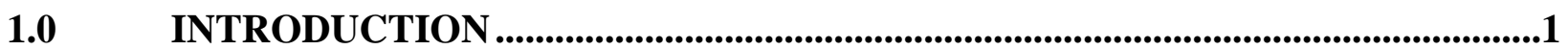

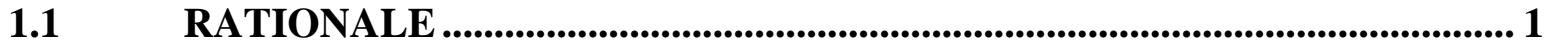

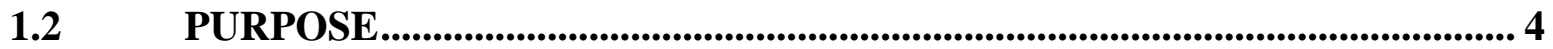

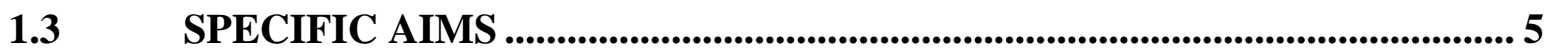

1.4 SIGNIFICANCE .............................................................................................. 5

2.0 REVIEW OF RELATED LITERATURE ............................................................7

2.1 THERMOREGULATION.................................................................... 7

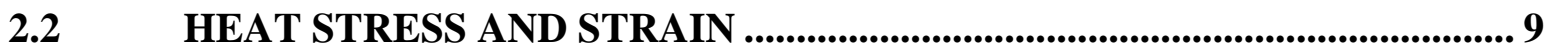

2.2.1 Heat Related Illness .............................................................................................. 9

2.2.1.1 Athletes versus Emergency Responders ................................................ 11

2.2.1.2 Epidemiology and Risk Factors ......................................................... 12

2.2.2 Incidence of Heat Illness ..................................................................................... 14

2.2.3 Physiological Responses to Heat Stress ............................................................ 15

2.2.4 Perceptual or Cognitive Responses to Heat Stress ........................................ 16

$2.3 \quad$ ASSESSMENT OF HEAT STRESS .................................................................... 17

2.3.1 Thermometry ........................................................................................................... 18

2.3.2 Heat Stress Indices........................................................................................... 20

2.3.2.1 Rational Indices of Heat Stress ................................................................ 20

2.3.2.2 Direct Indices of Heat Stress .......................................................... 22

2.3.2.3 Empirical Indices of Heat Stress.............................................................. 25 
2.4 PERCEPTUAL HEAT STRAIN INDICES ........................................... 25

2.5 SUMMARY OF LITERATURE REVIEW ................................................ 27

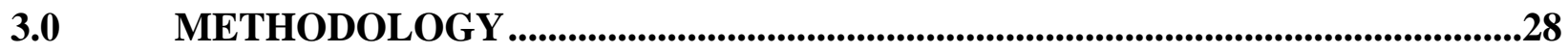

EXPERIMENTAL DESIGN _........................................................................ 29

3.1.1 Subjects.......................................................................................................... 30

3.1.2 Baseline Session.................................................................................... 31

3.1.3 Fifty Minute Exercise Sessions ................................................................. 32

3.1.4 Physiological Measures ................................................................................. 33

3.1.5 Perceptual Measures ........................................................................ 34

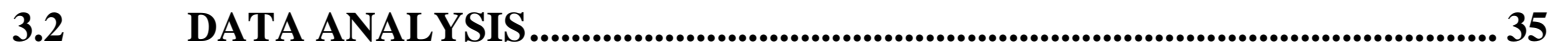

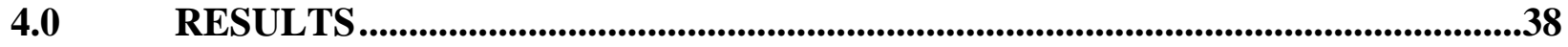

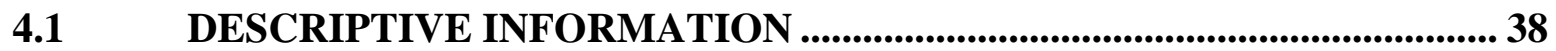

4.1.1 Analysis of Homogeneity ...................................................................... 39

4.1.1.1 Body Core Temperature............................................................................... 39

4.1.1.2 Heart Rate...................................................................................... 40

4.1.1.3 Ratings of Perceived Exertion.......................................................... 41

4.1.1.4 Ratings of Thermal Sensation.................................................................. 41

4.1.2 Relation between Physiological and Perceptual Responses...................... 42

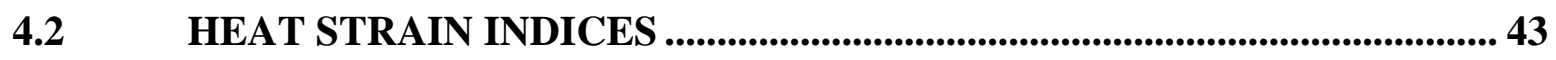

4.2.1 Physiological Heat Strain Index ..................................................................... 43

4.2.2 Perceptual Heat Strain Index ............................................................... 44

4.3 DEVELOPMENT OF THE PHI.............................................................. 45

5.0 DISCUSSION, CONCLUSION, AND RECOMMENDATIONS .........................49

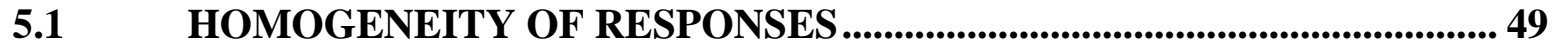

5.2 PHYSIOLOGICAL RESPONSES............................................................... 51

PERCEPTUAL RESPONSES ........................................................... 52

5.4 RELATION BETWEEN PHYSIOLOGICAL AND PERCEPTUAL

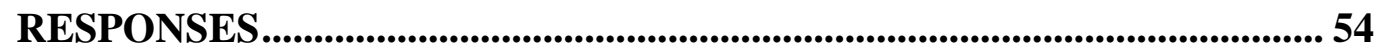

5.5 THE PERCEPTUAL HYPERTHERMIA INDEX .................................. 56

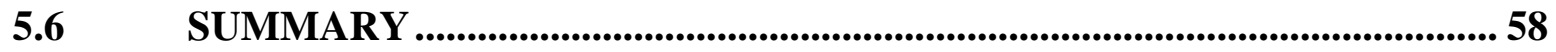

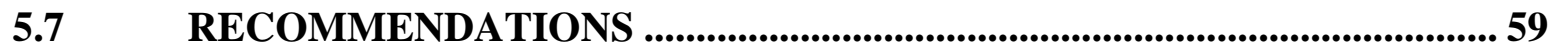


5.7.1 Future Research - Experimental Design...................................................... 59

5.7.2 Future Research - PHI Applications .......................................................6 60

APPENDIX A: METABOLIC RATE CATEGORIES ................................................63

APPENDIX B: STANDARDIZED INSTRUCTIONS FOR PERCEPTUAL

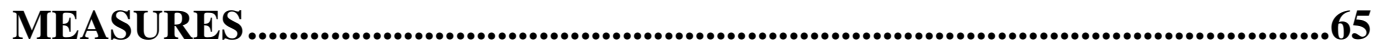

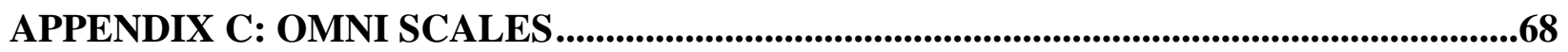

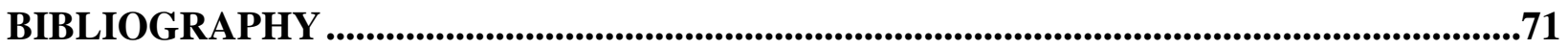




\section{LIST OF TABLES}

Table 1. Screening Criteria for Heat Stress Exposure (WBGT values in $\left.{ }^{\circ} \mathrm{C}\right)$.............................23

Table 2. Problem statements for the four interrelated investigations of thermal stress used in the present study. .28

Table 3. Descriptive characteristics of the combined sample and the four interrelated investigations. Mean \pm SD

Table 4. Levels of exertional heat strain associated with the calculated PSI and the corresponding measured $\mathrm{T}_{\text {re }}$ matched with the colored zones for the newly developed PHI

Table 5. Physiological and perceptual responses during treadmill exercise in the heat.

Mean \pm SD

Table 6. Correlation coefficients between body core temperature $\left(\mathrm{T}_{\mathrm{c}}\right)$, ratings of perceived exertion (RPE), and ratings of thermal sensation (RTS).

Table 7. Comparison of body core temperature between those reported by Moran et al. and those measured in the present investigation. Mean \pm SD

Table 8 . Body core temperatures $\left(\mathrm{T}_{\mathrm{c}}\right)$ for each calculated perceptual strain rating. Mean \pm SD.

Table 9. Mean body core temperature ranges for the Perceptual Hyperthermia Index (PHI)......47 


\section{LIST OF FIGURES}

Figure 1. Experimental designs across the four interrelated investigations. Data will be taken prior to starting exercise and at the conclusion of the initial 20-min exercise block (hyphenated rectangle) and at test termination.

Figure 2. A Hypothetical PHI. Areas of high RPE with low RTS and low RPE with high RTS (hyphenated circles) that are mathematically estimated using PeSI. The solid arrow represents the increase in body core temperature with increases in RPE and RTS.

Figure 3. First stage development of the PHI with perceptual strain index rating presented for concurrent rating of perceived exertion and thermal sensation. Values in each cell are perceptual strain index ratings.

Figure 4. Second stage development of the PHI. PeSI ratings were replaced with the concurrent mean body core temperatures. Mean body core temperatures presented in each cell were identified by measured ratings of perceived exertion and thermal sensation.

Figure 5. Third stage development superimposes the PHI color scheme over the mean body core temperatures (as presented by each cell) for a given PeSI.................................48

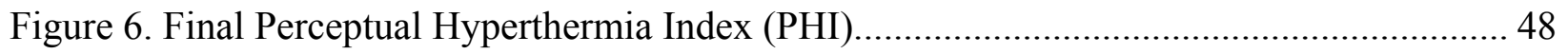

Figure 7. Proposed nine discernable sectors of the PHI to examine separately in future investigations. 


\section{PREFACE}

The author would like to extend the most sincere appreciation to the following individuals:

- Dr. Robert J. Robertson for his constant support, mentoring, and guidance in my professional development throughout my entire graduate educational experience.

- Drs. Elizabeth F. Nagle, Fredric L. Goss, and Mark A. Schafer for serving on my committee, and also for all their support over the years inside and outside of the classroom.

- David Hostler for his mentoring and generosity in allowing me the opportunity to work with him and everyone else at the Emergency Responder Human Performance Laboratory.

- Jamey Bednez, Sarah Kerin, Julia Morley and Gillian Beauchamp for their assistance in data collection. 


\subsection{INTRODUCTION}

This investigation developed a Perceptual Hyperthermia Index (PHI) to evaluate exertional heat strain during treadmill exercise. It was expected that a standardized index could be developed to measure hyperthermia using ratings of perceived exertion (RPE) and ratings of thermal sensation (RTS). Data were derived from both firefighters and healthy adult volunteers who performed bouts of treadmill exercise. The development of a hyperthermia metric provides a fast and easy assessment of exertional heat strain in firefighters during fire suppression and rescue operations.

\subsection{RATIONALE}

Fire suppression and rescue is physiologically very demanding on firefighters due to the extreme hot environmental conditions as well as the additional physical and thermal burden of the firefighting gear. The hot environment imposes a challenge on the homeostasis of body

temperature inducing hyperthermia. ${ }^{4,15,43,56}$ Additionally, the increased metabolic demand associated with physical movement while wearing firefighting thermal protective clothing (TPC) further increases body core temperature..

The assessment of hyperthermia is complex, involving both physiological and perceptual strain indicators. Heat stress indices assess the combined contributions of metabolic cost, environmental factors and clothing requirements. Heat strain indices assess the physiological 
response resulting from heat stress. A number of indices have been constructed to measure the degree of heat stress imposed on an individual. These heat stress indices may be categorized into three groups: rational, empirical, and direct. ${ }^{19,45,55,61}$ Rational indices are based on a heat balance equation that includes heat storage rate, metabolic rate, external work rate, radiant heat exchange, convective heat exchange, respiratory heat exchange, and evaporative heat loss. The heat stress index $(\mathrm{HSI})^{2}$ and the physiological heat strain prediction model by McPherson ${ }^{45}$ are examples of rational indices. Direct indices are based on actual measures of environmental variables. Examples of such direct indices are the wet-bulb globe temperature (WGBT) ${ }^{78}$ and the discomfort index (DI ${ }^{68}$ both of which are used to quantify the degree of heat stress imposed on an individual in a given set of environmental conditions. Many health and safety standards associated with environmental heat stress use a direct approach. Lastly, empirical indices are based on objective and subjective strain. One such example of an empirical index based on objective measurements is the physiological strain index (PhSI), developed by Moran and colleagues. ${ }^{46,50}$ This index was intended to address measurement limitations associated with direct environmental indices. The empirical index incorporates heart rate and core temperature response to a thermal load as surrogate measures of heat stress imposed on the individual.

Epstein and Moran ${ }^{19}$ suggested that rational and empirical indices provide comprehensive assessments of thermoregulation. Direct indices, though, have the benefit of being "more friendly and applicable" but only consider environmental variables such as wet-bulb, dry-bulb, and black-bulb temperatures. However, Cheung ${ }^{14}$ proposed that occupational exposure standards should not only use physiological outcomes, but also thermal perceptions reflecting the behavioral and exercise response to heat stress. The majority of these safety standards and indices of thermal strain do not incorporate perceptual responses to heat stress and hyperthermia. 
One possible method for monitoring the risk of heat-related injury involves the use of RTS and RPE derived from category metrics. Tikusisis et al. ${ }^{70}$ incorporated these two measures into a perceptual strain index (PeSI).

Hostler et al. ${ }^{31}$ examined the effects of hyperhydration on emergency responders performing treadmill exercise while wearing chemical resistant personal protective equipment (PPE). As part of their investigation, PhSI and PeSI were calculated for each subject performing under experimentally induced thermal stress. The results indicated that pre-participation hyperhydration did not influence perceptual or physiological strain indices and that perceptual heat strain was significantly greater than the physiological heat strain for both the control and hyperhydrated conditions. The unequal ratings between perceptual and physiological heat strain in the presence of a similar heat stress load suggests factors other than body core temperature and heart rate may mediate perceived thermal strain. One such factor may be the individual's perception of physical exertion which can interact with the subjective indices of heat strain such as thermal sensation during exercise under hot ambient conditions. Perceived thermal strain may be a protective mechanism to prevent heat-related injury. As such, a perceptual heat strain index may provide a measure of relative risk for individuals performing exercise while wearing TPC.

Although the use of perceptual responses to assess thermal strain is not a novel concept, the suggestions made by Epstein and Moran $^{19}$ are important in bridging the gap between the ease and applicability of direct indices and the comprehensiveness of empirical indices. The next logical step in hyperthermia risk assessment consequent to firefighting while wearing TPC was to develop an occupational exertional heat strain metric. This metric incorporated components of the $\mathrm{PeSI}^{70}$ into a graphical presentation providing a fast and practical observation tool to assess exertional heat strain during actual fire scenarios. The PHI metric was based on a pilot 
investigation performed in the Emergency Responder Human Performance Laboratory as

reported by Hostler et al. ${ }^{31}$ These data demonstrated a moderately strong correlation between PeSI and body core temperature under varying environmental heat loads.

\subsection{PURPOSE}

The present investigation developed a perceptually based hyperthermia metric to measure physiologic exertional heat strain during treadmill exercise. Data to develop the hyperthermia metric were derived from four separate subject cohorts who performed a standard exercise forcing function under high environmental temperatures while wearing TPC. The subject cohorts were participants in four inter-related laboratory experiments examining various aspects of the hyperthermic response during exercise while wearing TPC. The development of a hyperthermia metric used RPE and RTS responses to treadmill exercise measured during the four laboratory experiments. The RPE and RTS values were incorporated into a graphical presentation forming the hyperthermia metric. Measures of body core temperature during exercise were used to establish concurrence between body thermoregulation and the degree of exertional heat strain predicted by the PHI. Measures of body core temperature during treadmill exercise were used to define the limits of each graphically depicted zone (strata), providing physiological documentation of the perceptually (PHI) categorized degree of exertional heat strain. 


\subsection{SPECIFIC AIMS}

Houghton et al. ${ }^{32}$ suggested that for a heat stress index to be applicable, it must meet four criteria: 1) the index should be measurable and accurate over a wide range of environmental and metabolic conditions, 2) the index should consider all important factors related to heat stress such as the environment, clothing, etc., 3) relevant measurements should reflect the worker's exposure without interfering with performance or activity, and 4) worker's exposure limits, as measured by the metric, should be reflected by physiological and/or psychological responses that indicate an increased risk to safety or health. Some components of these guidelines are impractical and beyond the scope of the current investigation such as examining responses over a wide range of environmental and metabolic conditions. Therefore, the outcomes of this study were to generalizable to volunteer adult firefighters and non-firefighters performing exercise in TPC. The specific aim of the present investigation was to develop the PHI using RPE and RTS to assess the level of heat burden during treadmill exercise in subjects wearing TPC.

\subsection{SIGNIFICANCE}

The development of a hyperthermia metric to assess the level of risk for exertional heat strain could provide a valuable field-based assessment for firefighters engaged in fire suppression and rescue. This metric may be beneficial in very high risk environments that threaten the lives of firefighters.

The application of a hyperthermia metric reflects the importance of monitoring the health and well-being of firefighters performing physiologically taxing duties associated with fire 
suppression and rescue while wearing protective clothing largely impermeable to ambient gases and particulates. 


\subsection{REVIEW OF RELATED LITERATURE}

\subsection{THERMOREGULATION}

Thermoregulation is the body's ability to maintain body core temperature around a set-point temperature of approximately $37^{\circ} \mathrm{C}$ with a normal range of $36.2^{\circ}$ to $37.7^{\circ} \mathrm{C} .^{13,42}$ Temperature regulation is critical to homeostatic function and is linked to thermogenesis (metabolic heat production). The goal of temperature regulation is to maintain this relatively narrow homeostatic temperature range for optimal physiological function. Heat balance is generally the sum of heat production and heat loss where changes in core temperature are the result of either an increase or a decrease in heat storage. The heat balance equation for the storage of heat $(\mathrm{S})$ takes into consideration the heat exchange pathways of metabolic heat production $(\mathrm{M})$, evaporation $(\mathrm{E})$, radiation $(\mathrm{R})$, convection $(\mathrm{C})$, and conduction $(\mathrm{K})$. Additionally, the heat balance equation may incorporate heat production from physical work or exercise (Work). The mathematical relation between these variables is described as $\mathrm{S}=$ Work $+\mathrm{M}-\mathrm{E} \pm \mathrm{R} \pm \mathrm{C} \pm \mathrm{K}$, where positive numbers indicate heat gain and negative numbers indicate heat loss. ${ }^{13}$

Heat production is primarily the result of metabolic heat production and physical work or exercise. Heat loss is the movement of heat down the thermal gradient from high to low temperature and primarily occurs by one of more of the following four processes of evaporation, radiation, convection, and conduction. Additionally, heat loss is highly dependent of the ambient temperature and humidity of environment. 
Evaporation is heat loss through the conversion of sweat as water on the skin into water vapor gas. This process accounts for approximately $25 \%$ of heat loss at rest but becomes a primary means of heat loss under most environmental conditions during exercise. Radiation accounts for approximately $60 \%$ of heat loss and is the indirect movement of heat in the form of infrared rays or electromagnetic waves. Heat may be lost due to skin temperature exceeding air temperature and heat may be gained due to direct exposure to sunlight but varies greatly by environmental condition. However, radiative heat loss is minimal during uncompensable heat grain found with exercise in thermal protective clothing. Convection is heat loss down the thermal gradient by the transference of heat from an object to surrounding liquid mediums such as moving water, air, and internal body fluids. During exercise, convection occurs as heat produced within the muscle is transferred to the surrounding interstitial fluids. Conduction is heat loss down the thermal gradient by transference of heat from two objects in direct contact with one another such as feet on the ground surface.

This complex coordination of thermoregulatory processes is primarily regulated by the preoptic area of the anterior hypothalamus. The preoptic area of the anterior hypothalamus is responsible for generating autonomic, endocrine, motor, and behavioral responses to an environmental stimulus ${ }^{8}$.

Hypothermia and hyperthermia manifest whenever the body is unable to maintain normal core temperature due to internal or external factors. Hypothermia is a collection of symptoms resulting from a decrease in core temperature due to the body's inability to increase thermogenesis to counter rapid heat loss. At the other end of the thermal spectrum is hyperthermia. Hyperthermia is a collection of symptoms resulting from an increase in core temperature due to the body's inability to adequately dissipate heat through increased sweating 
and skin blood flow in the presence of an uncompensable heat gain. Exercise, clothing, and ambient conditions may increase body core temperature exceeding the homeostatic upper limit.

\subsection{HEAT STRESS AND STRAIN}

Heat stress is defined as the "net heat load to which a worker may be exposed from the combined contributions of metabolic cost, environmental factors and clothing requirements". Heat strain is

defined as the "overall physiological response resulting from heat stress." ${ }^{11}$ Heat stress can be prevented whereas heat strain requires medical treatment. Heat stress assessments can be used to evaluate the risk of heat-related illness. ${ }^{1,11}$ During compensable heat stress, the thermoregulatory system is able to adjust for increases in body heat and thus maintain a physiologically safe core temperature. Uncompensable heat stress conditions can be encountered by emergency responders and soldiers performing work in chemical or thermal protective clothing. Under these conditions, the body is unable to adequately thermoregulate and core temperature increases to dangerous levels often associated with exhaustion. ${ }^{37}$

\subsubsection{Heat Related Illness}

As heat stress surpasses the body's ability to maintain core temperature, the risk of heat-related illness increases. Heat-illness can be divided into two classifications based upon exposure time and intensity of the heat-stress. Minor heat-illnesses include heat cramps and heat syncope. Heat cramps are characterized as intense muscle spasms in the legs, arms, and abdomen resulting from fluid and sodium deficits. Heat syncope is fainting/dizziness during exposure to heat stress resulting from the pooling of blood in the venous circulation of the skin and leg muscles. This 
occurs most commonly during dehydration but may be the first symptom of heat exhaustion. Both heat cramps and heat syncope most commonly occur in an individual who is not heat acclimatized.

Major heat-illnesses include heat exhaustion and heat stroke; respectively in order of severity. Heat exhaustion is characterized as a mild to moderate illness due to the inability to sustain cardiac output and moderate to high core body temperatures $\left(38.5^{\circ} \mathrm{C} \text { to }>40^{\circ} \mathrm{C}\right)^{11,13}$ usually accompanied by wet or clammy skin. Signs of possible heat exhaustion include a rapid pulse, headache, syncope, nausea/vomiting, muscle cramps, and chills/goosebumps.

Heat stroke is characterized by central nervous system dysfunction and extremely high core body temperatures $\left(>40.5^{\circ} \mathrm{C}\right) \cdot{ }^{7,11,13}$ Signs of possible heat stroke include a weak or rapid pulse, changes to the central nervous system (confusion, agitation, apathy, delirium, and convulsions), hot and wet or dry skin (depending of the variant of heat stroke), vomiting, and hyperventilation. ${ }^{5,11,17}$ There are two types of heat stroke. Classical heat stroke results from extended exposure to hot environments that may prevent dissipation of body heat, typically occurring during summer heat waves. In these instances, individuals may stop sweating and evidence hot and dry skin. Exertional heat stroke occurs from increased metabolic demands of physical activity (i.e. running) that is sometimes undertaken in hot environments. The total produced heat load exceeds the body's maximal heat dissipation capacity with the individual typically experiencing hot and wet skin due to the enclosed thermal environment. ${ }^{13}$ Exertional heat exhaustion and heat stroke are of concern for firefighters and emergency personnel wearing thermal protective clothing or hazardous material personal protective equipment. 


\subsubsection{Athletes versus Emergency Responders}

Many thermoregulatory responses to heat stress are similar between athletes performing under high ambient temperature and humidity, and emergency responders performing work in thermal protective clothing. However, the factors that bring about heat strain may be different depending on the exercise to be performed.

Much of the heat stress literature involving athletic performance primarily deals with the sports of football and endurance events such as marathon running. Trained athletes participating in competition or a conditioning session perform exercise at a target intensity level for a specified duration. Marathon runners will run a sustained performance at a moderate intensity over the full 26.2 miles requiring approximately two to three hours at the elite level and upwards of 5 hours for the novice. Football players will participate in alactic exercise of short, intense bouts of exercise wearing protective clothing that contributes minimally to the thermal load. In short, heat stress during athletic performance is mostly associated with the environment (i.e. temperature and humidity) and metabolic heat production during exercise as energy requirements of muscular action increase. Athletic clothing is normally not a factor in provoking heat stress.

Emergency responders performing work while wearing chemical or thermal protective clothing are at risk for thermal strain. The protective clothing effects heat exchange by thermal insulation and evaporative resistance. ${ }^{30}$ Thermal insulation and evaporative resistance increases

the physiological and psychological strain on the wearer. ${ }^{44}$ These conditions can occur during both prolonged light work and shorter bursts of high intensity work. Ambient conditions may impose another strain on the wearer in addition to that of the protective clothing. 


\subsubsection{Epidemiology and Risk Factors}

Exertional heat stress does not similarly affect all individuals. As with any illness, there are predisposing risk factors and gradations of symptoms. These risk factors may be divided into four categories: individual characteristics, health conditions, medications, and environmental factors. ${ }^{1,11,13}$

Individual characteristics that may increase the likelihood and severity of exertional heat stress or strain include age, gender, a low physical fitness level, excessive body weight, lack of heat acclimatization, and dehydration. The thermoregulatory response does not differ between adults and children until very hot ambient temperatures are encountered $\left(45^{\circ} \mathrm{C}\right)$. Under these environmental conditions, children have a lower heat tolerance than adults primarily due to a higher body surface area to mass ratio. Although a high surface area to mass ratio is generally an advantage in thermoregulation, this advantage becomes a risk when the environmental temperature exceeds skin temperature causing the skin to absorb heat. ${ }^{11}$ There is some evidence that males and females do not differ in response to exertional heat stress when the genders have similar cardiorespiratory fitness levels and body composition. However, effects of gender are often difficult to ascertain in part by the presence of the confounding variables of heat acclimatization and aerobic fitness levels. When males and females perform at the same absolute exercise intensity, females exhibited a higher core temperature than males. This response is primarily due to females on average having a lower $\mathrm{VO}_{2 \max }$ than men. As such, a given absolute exercise intensity represents a higher relative aerobic metabolic rate for females. ${ }^{1,11}$ Studies examining gender differences should be interpreted with caution as basal body core temperature is different during the various phases of menstruation. Low aerobic fitness level, which can vary with age and gender, is also a risk factor for heat related illness. Thermoregulatory differences between children and older adults are often due to differences in the level of physical fitness. It is 
presumed that older adults will have decreased physical function and increased physical impairments. However, when adults are matched on aerobic fitness level using $\mathrm{VO}_{2 \max }$, the cardiovascular and thermoregulatory strain was independent of age. ${ }^{1,11}$

In many individuals, a low level of physical fitness is accompanied by higher than normal body weight. Excess body weight is evidenced by a high body mass index (BMI) and body fat percent (obesity). Gardner et al. ${ }^{24}$ found that in Marine Corps recruits, a comparatively high BMI $\left(\geq 22 \mathrm{~kg} / \mathrm{m}^{2}\right)$ or a slower 1.5 mile run time ( $\left.\geq 12 \mathrm{~min}\right)$ increased the risk for exertional heat illness by three-fold. In addition, the recruits with both poor fitness and higher weight increased their risk of heat illness by eight-fold. However, many obese individuals perform just as well as lean individuals under heat stress. This could be attributed to a form of heat acclimatization that occurs in obese individuals secondary to the insulating effect of peripheral body fat. Wallace et al. ${ }^{76}$ indirectly support this notion as after 14 weeks of basic training for Marine Corps recruits, only a slow run time was a predictor of exertional heat illness. Initially a high BMI was a significant predictor variable in statistical models to estimate heat illness. After training, a high BMI was no longer a predictor in the statistical model due to conditioning induced reduction in fat mass and increased heat acclimatization.

Heat acclimatization is one of the more important factors in reducing risk of or the severity of heat-related illness. Heat acclimatization involves a series of biological adjustments to compensate for the adverse physiological effects of heat stress. Exposure to a hot environment over a 10-14 day period (acute acclimatization) or months to years (adapted acclimatization) will improve comfort and safety, and exercise performance under hot environments. Prolonged exposure to heat of at least 100 to 120 minutes is necessary to elevate core and skin temperature to induce sweating is necessary to acclimate to the heat. ${ }^{13}$ The benefits of heat acclimatization 
are two-fold: minimize heat strain and improve exercise performance. Heat strain is minimized due to a reduction in core temperature that is the byproduct of improved sweating (earlier onset and higher rate), improved skin blood flow (earlier onset and higher rate), and a lowered metabolic rate. ${ }^{1,11,13}$ Exercise performance is improved due to an improvement in cardiovascular stability that is the product of lowered heart rates, increased stroke volume, maintained blood pressure, reduced sodium losses in sweat and urine, and increased plasma volume. In comparison to the non-acclimatized individual, the acclimatized individual has better evaporative, radiative, and convective heat loss.

Another factor of equal importance to risk of heat related illness is hydration status. Dehydration negatively influences exercise performance and seriously limits the adjustment to heat. When exercising in a hypohydrated condition, the onset of sweating is delayed until a higher core temperature is obtained, skin blood flow is reduced, and heart rate is increased in an attempt to compensate. Thermoregulation is compromised by the reduction in blood volume and the increase in hematocrit which increases blood viscosity. As blood viscosity increases, the convective distribution of heat is reduced increasing body heat storage and increasing core temperature. The advantages of heat acclimatization and high fitness level are lost when exercising in a hypohydrated condition. ${ }^{13}$

\subsubsection{Incidence of Heat Illness}

Bonauto et al. ${ }^{6}$ examined the incidence of heat related illness among non-military working populations in Washington State from 1995 to 2005. During that 11-year span, there were 480 workers' compensation claims for heat related illness, of which $78.5 \%$ occurred during outdoor work. The average annual insurance claim for heat related illness in Fire Protection was 80.8 per 
100,000 full-time equivalent (FTE). The annual claim for Fire Protection was higher than roofing construction (59.0 per 100,000 FTE), and highway, street, and bridge construction (44.8 per 100,000 FTE). For employment purposes, FTE is defined by the Federal Government Accountability Office as the number of total hours worked divided by the maximum number of compensable hours in a work year as defined by law. If the work year is defined as 2,080 hours, then one worker occupying a paid full time job all year would account for one FTE. Two employees working 1,040 hours each would consume one FTE between the two of them. Additionally, the heat related illness claim rates in North America were highest in the third quarter of each year due to increased exposure to hot environments. The higher outdoor temperatures from May to September accounted for $95 \%$ of the total heat related illness claims during these months. During the third quarter (July through September) alone, the highest rates of heat related illness claims were for roofing construction at 161.2 per 100,000 FTE and fire protection at 158.8 per 100,000 FTE.

\subsubsection{Physiological Responses to Heat Stress}

Cerebral changes during exercise in the heat may contribute to "central fatigue". 53,54,57 The notion of "central fatigue" is supported by the observation that exercise-induced hyperthermia is associated with reduced voluntary activation of the alpha motor neurons during sustained maximal muscle contractions. ${ }^{58}$ Furthermore, a reduced level of central activation has also been observed during passive hyperthermia. ${ }^{51,71}$ Mechanisms for the decrease in muscular and cognitive performance may be due to the attainment of a critical high core temperature. ${ }^{53}$ Studies by Gandevia ${ }^{22}$ and Bigland-Ritchie et al. ${ }^{3}$ found that a high core temperature impaired the ability to maintain maximal muscle activation resulting in a reduction of force generated. The reduced 
force generated seems to relate to reduced motor unit activation secondary to central nervous system fatigue. The combined effects of exercise and heat stress further exacerbate failure of the central nervous system by introducing competition for blood between the muscles, skin, and the brain. Although there is a reduction in cerebral blood flow, cerebral oxygen delivery does not appear to be jeopardized during exercise and thermally stressful conditions. ${ }^{59}$ One apparent consequence of reduced cerebral blood flow was a reduction of total body heat removal as there was not temperature gradient between the brain and the rest of the body due to increased core temperature.

\subsubsection{Perceptual or Cognitive Responses to Heat Stress}

Psychological responses to firefighting activities indicate potential impairments in cognitive function. ${ }^{35,66}$ Kivimaki and Lusa $^{35}$ found that task-focused thinking decreased as heat stress increased during smoke-diving simulations. Using the continuous performance test (CPT), a

computerized assessment of reaction time and accuracy, Smith et al.$^{66}$ similarly found that the accuracy of responding (number of errors) decreased following the first exposure to a live-fire trial. Initially, participants reduced the number of errors by $4.2 \%$ following trial one but made 4.6\% and $12.0 \%$ more errors following trials two and three, respectively. These data may indicate that cognition may decrease as result of extended work performance in thermally stressful situations. The underlying explanation of these findings is the combined negative effects of hyperthermia and dehydration on cognitive function. Such combined effects of hyperthermia and hypohydration during work performed by emergency responders while wearing thermal protective clothing may further exacerbate impairments in cognitive function. 
Maughan et al. ${ }^{42}$ summarizes in their review that performance on physical and mental tasks are significantly reduced by heat stress and dehydration. This response is due to the additional stress imposed on the cardiovascular system that may be a detriment to the central nervous system. Cheung ${ }^{14}$ described a fundamental problem with previous research that assessed brain activity during hyperthermia. They noted that few studies tracked the effect of thermal stress on cognitive and task performance impairments in the presence of concomitant changes in physiological and/or perceptual thermal strain. As part of their training, the Australian Defense Forces have been exposed to "debilitating" tropical environments that negatively impacts physiological performance. Hocking et al. ${ }^{28}$ observed that in addition to these physiological detriments, thermal strain did not impact cognition of those military personnel. It was found that even through subjects experienced increased cardiovascular strain, the psychometric test batteries showed no significant performance detriments yet there was a marked difference in the electrical responses of the brain when thermally strained. Cheung ${ }^{14}$ suggests that while cognitive impairment may be sensitive to thermal stress, it may also be negated or minimized by other compensatory mechanisms that limit performance degradation. This may help explain conflicting results among studies examining hyperthermia (and the resulting hypohydration) on cognitive function.

\subsection{ASSESSMENT OF HEAT STRESS}

Heat stress is measured directly and indirectly through thermometry and heat stress indices that may include environmental, physiological, and perceptual variables. 


\subsubsection{Thermometry}

Thermometry is the primary tool to measure core and skin temperature. There are a variety of thermometric instruments, each with their own strengths and weaknesses which can be categorized as invasive and noninvasive.

Invasive techniques include rectal, oesophageal, and ingestible thermistors. Rectal thermometry is considered the most practical and accurate for measuring core temperature and is accepted as the criterion for temperature measurement in hyperthermic athletes ${ }^{12}$ and for scientific research of heat exhaustion or stroke. ${ }^{48}$ Additionally, rectal temperature is recommended in the National Athletic Trainers Association's position statement regarding detection of thermal strain. ${ }^{4}$ However, rectal thermometry may have a prolonged response time compared with other techniques, thereby being relatively slow to capture rapid changes in core temperature. $^{48}$ One alternative to rectal thermometry is oesophageal temperature. The oesophagus is preferred by many to assess core temperature because of its location deep within the body juxtapositioned to the left ventricle and aorta. Therefore, it reflects the temperature of blood flow to the hypothalamus. ${ }^{48}$ Unlike rectal thermometry; oesophageal temperature has a rapid response but comes with high subject burden. The thermistor may be difficult to insert, may cause irritation to the nasal passages, and general subject discomfort ${ }^{65}$ and is not generally applicable in clinical settings. Another alternative to rectal thermometry is the use of ingestible thermistors that assess the core temperature within the gastrointestinal tract. Intestinal temperature has been shown to consistently record higher temperatures than rectal temperature when measured simultaneously during cycling exercise. ${ }^{36,38,67}$ However, intestinal temperature provided a better representation of rectal temperature than oesophageal temperature. ${ }^{60}$ Gant et al. $^{23}$ examined the validity and reliability of intestinal temperature during intermittent running. 
The results suggested that the test-retest variability of intestinal temperature was acceptably small during intermittent shuttle running. The small random measurement error and similar thermal responses to exercise suggest that intestinal temperature is appropriate for use in research as an alternative to rectal temperature. Additionally, Casa et al. ${ }^{12}$ found that intestinal temperature had a correlation coefficient of 0.86 with rectal temperature and a mean bias of $0.19^{\circ} \mathrm{C}$. The authors suggested that intestinal temperature provided a valid indication of the body temperature rise and fall associated with the onset and cessation of exercise performed in higher ambient temperatures.

Noninvasive thermometric techniques typically include oral, aural (tympanic), and the temporal artery measurements. Oral thermometry is easy accessible and changes quickly in correspondence with changes in core temperature. Oral thermometry may be affected by eating, drinking, breathing, swallowing, facial fanning, and air temperature. ${ }^{12,48}$ Oral temperature during exercise and post-exercise has been shown to be consistently lower than rectal temperature. In addition, it has been suggested that oral temperature is not be a valid measure to assess hyperthermia in exercising individuals. ${ }^{12}$ Aural temperature is assessed in the ear canal and is easy to use. ${ }^{48}$ The actual temperature that is assessed is the average of the heat in the tympanic membrane, the air within the ear canal, and that radiated from the inner canal wall. ${ }^{12}$ Devices that assess aural temperature use conversions to improve accuracy as aural temperature is often lower than the more established measures of core temperature. Despite these conversions, aural temperature has been shown to be consistently lower than rectal temperature. This may be attributed to the low sensitivity of aural temperature to detect internal temperature changes resulting from alterations in blood flow to the skin, air or sweat evaporative cooling, and moisture or sweat in the ear canal. ${ }^{12}$ Temporal thermometry is a relatively new, easy to use 
device that scans the temporal artery. ${ }^{41}$ Studies examining temporal thermometry have found it to be a valid instrument during resting conditions ${ }^{25,26}$ but not during indoor exercise in the heat..$^{34,41}$ Temporal temperature was also found to be significantly lower than rectal temperature before, during, and after outdoor exercise in the heat. ${ }^{12}$

\subsubsection{Heat Stress Indices}

A heat stress index is a single value that integrates the effects of any thermal environment that will vary with the thermal strain experienced by an individual. ${ }^{17}$ All heat stress indices are based on one or more of the following parameters: sweat rate, heart rate, core temperature, environmental condition. ${ }^{9}$ Heat stress indices go back over one hundred years to Haldane's suggestion in 1905 that wet-bulb temperature is the most appropriate measure to express heat stress. ${ }^{27}$ Since that time, there have been a large number of indices developed and used throughout the world. ${ }^{17}$

Heat stress indices can be divided into three groups according to their rationale and function: rational indices, direct indices, and empirical indices.

\subsubsection{Rational Indices of Heat Stress}

Rational indices are the most comprehensive of the three groups of heat stress indices. These indices integrate environmental and behavioral variables and are based on calculations involving the heat balance equation. ${ }^{17}$ In general; these indices are considered impractical with some parameters entered as a constant in the calculations. Examples of rational indices include the operative temperature, the Belding-Hatch Heat-Stress Index, and skin wettedness. 
The operative temperature index was derived by Winslow et al. ${ }^{77}$ to express heat exchange between a worker and the surrounding environment by radiation and convection. The operative temperature is derived from the heat-balance equation where the combined effect of radiation and convection is a weighted sum of the heat-transfer coefficients. However, in order to derive the operative temperature, skin temperature must be measured or assumed and air velocity must also be measured. Humidity and metabolic heat production were omitted making generalizability limited. ${ }^{55}$

The Belding-Hatch Heat-Stress Index (BH-HSI) ${ }^{2}$ has been used widely in laboratory and field settings for heat stress studies. It appears in a table format that identifies physiological and psychological consequences of exposure time to thermal stress. The BH-HSI is a derivation of the heat-balance equation that includes environmental and metabolic factors. It is a ratio calculated as the amount of body heat that is required to be lost to the environment by evaporation for thermal equilibrium divided by the maximum amount of sweat evaporation allowed through the clothing that can be accepted by the environment. ${ }^{55}$ However, the BH-HSI assumes a constant sweat rate for everyone, a constant skin temperature of $35^{\circ} \mathrm{C}$, and conventional long-sleeved shirt and trouser ensemble. ${ }^{17,55}$

Skin wettedness indices are based on the concept that the efficiency of sweat evaporation will affect heat strain such that the less efficient the evaporation, the greater the surface area of the body that needs to be wetted with sweat to maintain evaporative heat transfer. These indices incorporates air temperature, humidity, air movement, radiative heat, metabolic heat, and clothing properties which all need to be measured or calculated for each situation. Due to these requirements, the skin wettedness indices are time consuming and impractical for routine environmental monitoring. ${ }^{55}$ 


\subsubsection{Direct Indices of Heat Stress}

Direct heat stress indices are based on environmental variables. These indices are commonly used to construct safety regulations by providing a simplified approach to obtain an estimate of thermal balance. ${ }^{17}$ These indices can either directly measure environmental variables to simulate heat strain or employ empirical calculations of environmental variables. Examples of direct indices include the dry bulb temperature, the wet bulb temperature, the effective temperature, the wet bulb globe temperature, and the discomfort index..$^{17,55}$

Dry bulb temperature is an easily measured estimate of comfort conditions for sedentary people wearing conventional clothing within a zone of light air movement, relative humidity of $20 \%$ to $60 \%$, and air temperatures of $22^{\circ} \mathrm{C}$ to $25.5^{\circ} \mathrm{C}$. The assumption of dry bulb temperature is that as work intensity increases the "comfort" air temperature decreases as a result of the heat balance equation. Wet bulb temperature is considered an appropriate metric where radiant temperature and air velocity are not large factors in assessing heat stress and predicting heat strain. Wet bulb temperature is easily measured and may be used in any hot, humid situation where wet bulb temperature approaches skin temperature, radiant heat is minimal, and air velocity is light. ${ }^{55}$

The Effective Temperature (ET) ${ }^{30}$ was developed by Houghton and Yaglou in 1923. The ET is an index based on direct measurements of environmental variables and is used to simulate heat strain. This index was initially developed to determine the relative effects of air temperature and humidity on comfort. ${ }^{17}$ The ET combines both the dry bulb and wet bulb temperatures, and air velocity. ${ }^{55}$ In 1932, Vernon and Warner ${ }^{74}$ created a corrected effective temperature (CET) to account for the effects of radiation by using black globe temperature instead of dry bulb temperature. The ET and CET have been widely used in studies of physical, psychomotor, and mental performance changes as a result of heat stress. The World Health Organization 
recommended that values do not exceed $30^{\circ} \mathrm{C}$ for sedentary activities, with these critical values decreasing as work intensity increases. ${ }^{55}$

The wet bulb globe temperature (WBGT) is now by far the most widely used heat stress index throughout the world. ${ }^{17}$ It was developed by the United States Navy in 1957 as a basis for environmental heat stress monitoring to control heat casualties during military training. ${ }^{78}$ The WBGT emerged from the CET and consists of weighting dry bulb temperature, wet bulb temperature, and black globe temperature thus combining the effects of humidity, air movement, radiation, and outside air temperature. ${ }^{55}$ The coefficients were determined empirically and the index has no physiological correlates. It was found that heat casualties and time lost due to heat stress were both reduced by using WBGT to set exposure limits. ${ }^{17}$ The WBGT criterion (Table 1) incorporates acclimatization state, metabolic rate category for the work (Appendix A), and the proportion of work within an hour. ${ }^{1}$

Table 1. Screening Criteria for Heat Stress Exposure (WBGT values in ${ }^{\circ} \mathrm{C}$ )

\begin{tabular}{|c|c|c|c|c|c|c|c|c|}
\hline \multirow[b]{2}{*}{ Work Demands } & \multicolumn{4}{|c|}{ Acclimatized } & \multicolumn{4}{|c|}{ Unacclimatized } \\
\hline & Light & Moderate & Heavy & Very Heavy & Light & Moderate & Heavy & Very Heavy \\
\hline $100 \%$ Work & 29.5 & 27.5 & 26 & & 27.5 & 25 & 22.5 & \\
\hline $\begin{array}{l}75 \% \text { Work; } \\
25 \% \text { Rest }\end{array}$ & 30.5 & 28.5 & 27.5 & & 29 & 26.5 & 24.5 & \\
\hline $\begin{array}{l}50 \% \text { Work; } \\
50 \% \text { Rest }\end{array}$ & 31.5 & 29.5 & 28.5 & 27.5 & 30 & 28 & 26.5 & 25 \\
\hline $\begin{array}{l}25 \% \text { Work; } \\
75 \% \text { Rest }\end{array}$ & 32.5 & 31 & 30 & 29.5 & 31 & 29 & 28 & 26.5 \\
\hline
\end{tabular}

*ACGIH. (2001). Heat Stress and Strain. Cincinnati, OH: American Conference of Governmental Industrial Hygienists.

The WBGT was eventually adopted by the International Organization for Standardization (ISO) as a standard for exposing workers to hot environments as well as the American Conference of Government Industrial Hygienists, the Occupational Safety and Health Administration, the American Industrial Hygiene Association, and the American College of Sports Medicine. ${ }^{17}$ Although WBGT is a standard for many organizations, the index is limited in 
its applicability across a broad range of potential heat stress scenarios and environments due to the inconvenience of measuring black globe temperature.

Since black globe temperature is not easily measured and may be an inconvenience in assessing heat stress, Lind and Hellon ${ }^{40}$ proposed the Oxford Index. This is a simple direct index based on a weighted summation of wet bulb temperature and dry bulb temperature. The coefficients employed a high weight assigned to wet bulb temperature reflecting the reliance on sweat evaporation for thermoregulation in hyperthermic conditions. Physiological strain was demonstrated by showing a high correlation between rectal temperature and heart rate with exercise tolerance time. However, the index is not appropriate during significant thermal radiation. ${ }^{17}$ In order to broaden application to other environments, a number of similar indices were developed that adopted different weights for the temperature variables. These were found to correlate with WBGT having $\mathrm{r}^{2}$ values ranging from 0.930 to 0.967 . One form of the modified Oxford Index is the Discomfort Index (DI) developed by Thom. ${ }^{69}$ The DI produced similar values to the WBGT, having an $\mathrm{r}^{2}$ of $0.947 .{ }^{17}$

The Discomfort Index was highly correlated with ET, and sweat rate during rest and exercise. A number of studies have examined the Discomfort Index in a diversed population under differing climate conditions which only broadens its appeal for assessing heat stress. Particularly, DI can determine the heat load at any given time and can be summed to provide information on a daily, monthly, seasonal, or yearly basis. The Israel Defense Forces and the Israeli Ministry of Education adopted the DI to provide guidelines for exercising in the heat and associated need for fluid intake. ${ }^{17}$

More recently, an Environmental Stress Index (ESI) was developed and validated against WBGT. ${ }^{49}$ The ESI has been evaluated and refined for hot/dry and hot/wet climates. ${ }^{47}$ The ESI is 
based on measurements of ambient temperature, relative humidity, and solar radiation. These variables were chosen due to common usage, measurement ease, and fast response time. ${ }^{47}$ High correlation $\left(r^{2}>0.899\right)$ between ESI and WBGT for the 19 different geographic locations in Israel, and $\mathrm{r}^{2}$ of 0.985 and 0.982 for the hot/dry and hot/wet geographic locations were found. ${ }^{47}$ The evaluations of ESI under extreme climatic conditions provide support for its use as a substitute to the WBGT index during athletic and military activity conducted in the heat. ${ }^{47}$

\subsubsection{Empirical Indices of Heat Stress}

However, these "standard" indices (a) assume that all individuals respond similarly to a given level of external heat stress, (b) are highly conservative in estimating thermal response and tolerance in order to accommodate a wide range of individual responses, and (c) may be cumbersome to determine in real-time. ${ }^{14}$ Empirical indices are based on objective and subjective strain ${ }^{17}$ and may incorporate physiological and perceptual responses to increased heat stress that address several limitations of the "standard" heat stress indices. Examples of empirical indices include the Cumulative Heat Strain Index (CHSI), the Physiological Strain Index (PhSI), and the Perceptual Strain Index (PeSI).

\section{$2.4 \quad$ PERCEPTUAL HEAT STRAIN INDICES}

One methodological issue is how heat stress may be defined and ultimately perceived. Heat stress is perceived as either thermal comfort or thermal sensation. Thermal comfort typically relates to how relatively comfortable an individual feels in a thermally stressful condition. Perceptual indices of thermal comfort use a Likert format that may range from "Comfortable" to "Very Uncomfortable". ${ }^{20}$ Thermal sensation typically indicates how hot or cold an individual 
feels in a thermally stressful condition and are presented in a bipolar format ranging from "Cold" to "Neutral" to "Hot". ${ }^{20}$ Some perceptual indices combine measures of comfort and sensation. As examples, the Bedford Thermal Scale employs descriptors that range from "Much Too Cool" to "Comfortable" to "Much Too Warm" while the OMNI Thermal Sensation Scale employs descriptors "Comfortable" to "Very Hot." Additionally, visual analog scales may also assess thermal perception ${ }^{39}$ that may increase sensitivity to changes in non-uniform conditions on the body surface. In essence, the visual analog scale is the developmental precursor or semantic differential for many perceptual indices such as the OMNI perceived exertion and muscle pain scales. Mower ${ }^{52}$ proposed that thermal sensation may be determined in part by the regional thermo-sensitivity independent of core temperature, and that thermal comfort may be linked to effects of sweating rate and skin wetness.

The concurrent validity of perceptual indices has been undertaken using physiological criteria. In the case of ratings of perceived exertion, oxygen consumption and heart rate are the two primary criterion physiological variables. Additionally, ratings of perceived exertion, although not a measure of thermal perception, do increase with a rise in core temperature. ${ }^{53,58}$ Perceived exertion during exercise with progressive hyperthermia has also been shown to correlate with reductions in middle cerebral artery mean blood velocity and electroencephalographic changes. ${ }^{58}$

Specifically, perceptions of thermal stress may be derived from the ambient environmental conditions, skin temperature, core temperature, and heart rate. Thermal comfort in helicopter aircrew wearing immersion suits seemed to show an anecdotal positive trend with ambient temperature, cabin temperature, and the cabin wet-bulb globe temperature index. ${ }^{18}$ 
Thermal sensation during exercise in cool and cold water is suggested to be moderately correlated with skin temperature and rectal temperature. ${ }^{72}$

\subsection{SUMMARY OF LITERATURE REVIEW}

In summary, the current knowledge base regarding temperature regulation and heat stress indices indicated the need to develop user friendly methods to quickly and accurately assess heat stress in thermally stressful conditions. These conditions may be imposed by the ambient environmental temperature or through the clothing properties (insulation, water vapor permeability) of thermal protective clothing and personal protective equipment that are often worn by emergency personnel. One possible means to assess homeostatic disruption of temperature regulation in firefighters is to develop a single, easy to apply index of thermal strain that employs concurrently reported measures of perceived exertion and thermal sensation. 


\subsection{METHODOLOGY}

The PHI was developed using RPE and RTS data derived from the following four interrelated investigations involving exercise induced thermal stress: (a) FIRE I (a laboratory study on rehydration), (b) FIRE II (a laboratory study of cooling), (c) COG I (a laboratory study of cognitive function), and (d) COG II (a laboratory study of cognitive recovery) (Table 2). Each of the four interrelated investigations employed partially overlapping subject cohorts performing a treadmill exercise forcing function under hot ambient conditions. During exercise, subjects wore the same type of TPC. The two FIRE trials are funded by the Federal Emergency Management Agency (FEMA) Assistance to Firefighters Grants (AFG) program.

Table 2. Problem statements for the four interrelated investigations of thermal stress used in the present study.

\begin{tabular}{|l|c|c|c|}
\hline Investigation & $\begin{array}{c}\mathbf{N} \\
\mathbf{( M / F )}\end{array}$ & Problem Statement \\
\hline FIRE I & $16 / 2$ & $\begin{array}{l}\text { To examine the effect of rehydration strategies of water, sport drink, and } \\
\text { intravenous normal saline on the duration of exercise, heart rate response, and } \\
\text { temperature response during a subsequent bout of exercise in TPC. }\end{array}$ \\
\hline FIRE II & $13 / 4$ & $\begin{array}{l}\text { To examine the effect of six cooling strategies on duration of exercise, heart } \\
\text { rate response, and temperature response during a subsequent bout of exercise } \\
\text { in TPC. }\end{array}$ \\
\hline COG I & $10 / 0$ & $\begin{array}{l}\text { To identify if a battery of cognitive tests can be used to quantify negative } \\
\text { changes in cognitive function following treadmill exercise in TPC. }\end{array}$ \\
\hline COG II & $14 / 5$ & $\begin{array}{l}\text { To document the recovery of cognition after performing strenuous work while } \\
\text { wearing firefighter protective clothing and equipment. } \\
\text { Secondary aims are to, 1) identify the effect of thermal stress on activation of } \\
\text { coagulation and inflammation and 2) examine possible loss and recovery of } \\
\text { functional balance following treadmill exercise in TPC. }\end{array}$ \\
\hline
\end{tabular}




\subsection{EXPERIMENTAL DESIGN}

All four interrelated investigations employed similar experimental designs with slight procedural differences between experiments (Figure 1). In all four protocols, RPE and RTS data were derived prior to starting exercise, following twenty minutes of treadmill walking at 4.5 kilometers per hour $\left(\mathrm{km} \cdot \mathrm{hr}^{-1}\right)$ and at test termination. Methodological commonalities and differences specific to each investigation are addressed below.

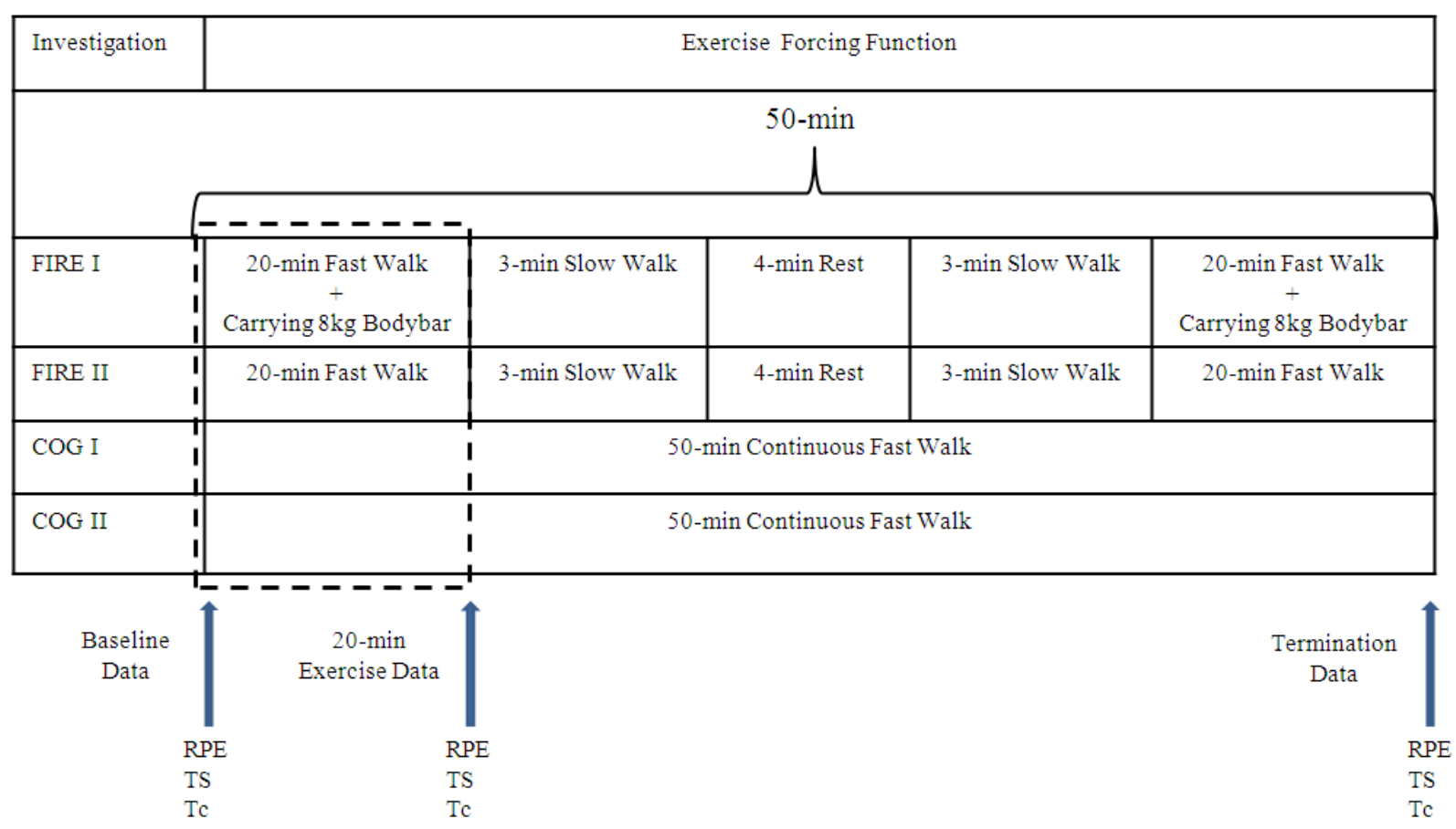

Figure 1. Experimental designs across the four interrelated investigations. Data will be taken prior to starting exercise and at the conclusion of the initial 20-min exercise block (hyphenated rectangle) and at test termination. 


\subsubsection{Subjects}

A total of sixty-five female $(n=11)$ and male $(n=54)$ adults aged 19 to 44 years old who comprised the experimental cohorts for the four investigations were subjects in this investigation (Table 3).

Table 3. Descriptive characteristics of the combined sample and the four interrelated investigations.

Mean \pm SD

\begin{tabular}{lccccc}
\hline & $\begin{array}{c}\text { Combined } \\
(\mathbf{N}=\mathbf{6 5})\end{array}$ & $\begin{array}{c}\text { FIRE I } \\
(\mathbf{n = 1 8})\end{array}$ & $\begin{array}{c}\text { FIRE II } \\
(\mathbf{n}=\mathbf{1 8})\end{array}$ & $\begin{array}{c}\text { COG I } \\
(\mathbf{n = 1 0})\end{array}$ & $\begin{array}{c}\text { COG II } \\
(\mathbf{n = 1 9 )}\end{array}$ \\
\hline $\begin{array}{l}\text { Age } \\
(\mathrm{yrs})\end{array}$ & $28.88 \pm 6.75$ & $29.89 \pm 8.65$ & $29.78 \pm 7.38$ & $28.10 \pm 5.34$ & $27.47 \pm 4.61$ \\
$\begin{array}{l}\text { Height } \\
(\mathrm{cm})\end{array}$ & $173.72 \pm 8.96$ & $173.11 \pm 9.57$ & $172.08 \pm 9.31$ & $175.10 \pm 6.35$ & $175.13 \pm 9.51$ \\
$\begin{array}{l}\text { Weight } \\
(\mathrm{kg})\end{array}$ & $78.34 \pm 15.99$ & $89.72 \pm 15.81$ & $75.38 \pm 14.43$ & $73.20 \pm 8.04$ & $73.05 \pm 16.17$ \\
$\begin{array}{l}\text { VO } \\
\left(\mathrm{ml} \cdot \mathrm{kg}^{-1} \cdot \mathrm{min}^{-1}\right)\end{array}$ & $43.82 \pm 9.31$ & $37.77 \pm 3.87$ & $38.10 \pm 6.91$ & $54.52 \pm 6.70$ & $49.33 \pm 7.87$ \\
$\begin{array}{l}\text { Body Composition } \\
(\% \text { Fat })\end{array}$ & - & $20.44 \pm 5.20$ & $15.08 \pm 25.13$ & -- & - \\
$\begin{array}{l}\text { BMI } \\
\left(\mathrm{kg} \cdot \mathrm{m}^{-2}\right)\end{array}$ & $25.74 \pm 5.06$ & $30.11 \pm 5.42$ & $25.13 \pm 3.97$ & $23.97 \pm 3.40$ & $23.09 \pm 3.74$ \\
\hline
\end{tabular}

All subjects were clinically healthy and had no prior experience in rating their perception of physical exertion. Subjects were recruited through letters sent to fire departments in Allegheny and adjacent counties and recruitment flyers posted throughout the University of Pittsburgh and surrounding areas. Interested individuals were asked to contact the Emergency Responder Human Performance Laboratory to schedule an initial visit. In this initial visit, individuals provided informed consent and completed a survey of demographic information, medical history, and physical activity level. Potential subjects underwent a physician administered physical examination including a resting 12-lead ECG and a 12-lead ECG recorded during a graded exercise test. Both ECGs were interpreted by a cardiologist. Individuals with orthopedic, cardiovascular and/or metabolic contraindications to exercise participation (i.e. coronary artery disease, prior myocardial infarction, peripheral vascular disease, hypertension, chronic 
obstructive pulmonary disease and diabetes mellitus) or who were pregnant were excluded from participation. Additionally, individuals taking medications that were expected to alter physiological response to exercise (i.e. beta blockers) were excluded.

\subsubsection{Baseline Session}

Descriptive characteristics of the subjects were recorded including body height $(\mathrm{cm})$, body mass (kg), and body fat (\%). Body height was determined using a Healthometer Scale with an attached stadiometer (Sunbeam Products, Inc., Boca Raton, FL). Fat free mass was measured using the Jackson and Pollack 3 skinfold site equation. ${ }^{33}$ Skinfold measurements were obtained using Lange skinfold calipers. Aerobic fitness was assessed using a graded exercise test on either a cycle ergometer (FIRE I, FIRE II) or a treadmill (COG 1, COG II). Oxygen consumption $\left(\mathrm{VO}_{2}\right.$; liters per minute; STPD) was measured using an open-circuit respiratory-metabolic system (True Max 2400, Parvo Medics, Salt Lake City, UT) during each minute of the exercise protocol. Heart rate (Polar Electro, Finland) was measured from 45 to 60 seconds of each minute of the exercise protocol. $\mathrm{VO}_{2 \text { peak/max }}$ was established as the highest measured value that occurred when the subject was no longer able to perform the exercise test due to fatigue. Secondary criteria for peak/maximal measurement included a $\mathrm{VO}_{2}$ plateau (i.e. a change $<3.5 \mathrm{ml} \cdot \mathrm{kg}^{-1} \cdot \mathrm{min}^{-1}$ between contiguous stages at maximal intensity), a RER $\geq 1.2$, and a peak exercise heart rate \pm 5 beats. $\min ^{-1}$ of age-predicted maximal heart rate. 


\subsubsection{Fifty Minute Exercise Sessions}

The evening prior to each exercise testing session, subjects were provided a thermistor capsule (CorTemp; HQ, Inc., Palmetto, FL) and instructed to ingest the capsule eight hours before their scheduled exercise session. The thermistor transmitted body core temperature to a hand-held monitor during the experimental condition. The subjects were instructed to drink 20 ounces of water the night before the scheduled exercise session and to refrain from alcohol, caffeine, and nicotine use for 12 hours prior to the exercise session. Subjects in FIRE I and FIRE II consumed meal replacement bars and sport drink to standardize morning caloric intake equal to $40 \%$ of the basal metabolic rate for a moderately active adult. Upon arriving at the laboratory, subjects provided a urine sample for a urine specific gravity (USG) measurement to ensure they were euhydrated. In female subjects, this sample was also used for a urine pregnancy test. All of these tests were administered by an investigator.

Subjects were given an opportunity to void and then weighed in short pants (shorts and sport bra for females). A heart rate monitor strap was placed around the subject's chest. Subjects then put on a standardized turn-out uniform of cotton-poly pants and a cotton t-shirt. Standardized instructions that included definition, scale anchors, frequency of administration and answering any questions for the perceptual measures of RPE and TS were read to each subject immediately prior to beginning the 50-minute exercise session (Appendix B). Next, subjects donned firefighter thermal protective clothing (TPC; heavy pants, heavy coat, nomex hood, boots, helmet, and gloves). A self-contained breathing apparatus (SCBA) was positioned on their back. After the subjects fully donned the TPC, they were instructed to stand on the treadmill. Baseline measures of heart rate, body core temperature, and perceptual measures were assessed at this time. 
Subjects in FIRE I and FIRE II performed an interval treadmill protocol in a heated interior room $\left(98-100^{\circ} \mathrm{F}\right)$ to simulate a fire suppression environment. This interval protocol consisted of 1) walking for 20 minutes at $4.5 \mathrm{~km} \cdot \mathrm{hr}^{-1}\left(2.8 \mathrm{mi} \cdot \mathrm{hr}^{-1}\right)$ at an incline of $\left.2.5 \%, 2\right)$ walking for three minutes at $\left.2.6 \mathrm{~km} \cdot \mathrm{hr}^{-1}\left(1.6 \mathrm{mi} \cdot \mathrm{hr}^{-1}\right), 3\right)$ standing at rest for four minutes, 4) walking for three minutes at $2.6 \mathrm{~km} \cdot \mathrm{hr}^{-1}$, and 5) walking for 20 minutes at $4.5 \mathrm{~km} \cdot \mathrm{hr}^{-1}$ at an incline of $2.5 \%$. With the exception of carrying an $8.2 \mathrm{~kg}$ bodybar in FIRE I, this 50-min interval protocol was the same for both FIRE I and FIRE II investigations (see Table 1). Subjects in FIRE I performed the interval protocol on three separate occasions and subjects in FIRE II performed the interval protocol on seven separate occasions. Subjects in COG I and COG II performed a 50 -min continuous treadmill protocol at $4.5 \mathrm{~km} \cdot \mathrm{hr}^{-1}$ in a heated interior room $\left(98-100^{\circ} \mathrm{F}\right)$. Termination criteria for the exercise session were a) at the subject's request (i.e. fatigue), b) a body core temperature $>39.5^{\circ} \mathrm{C}, \mathrm{c}$ ) $\mathrm{HR}>10$ beats $\cdot \mathrm{min}^{-1}$ of age-adjusted $\mathrm{HR}_{\max }$, or d) undertaken at the investigators discretion for the subject's safety (i.e. unsteady gait). Heart rate and core body temperature were measured every 2 minutes of the exercise protocol. RPE and RTS measured at the conclusion of the initial 20-min exercise and again at test termination will be used in the present investigation to develop the PHI.

\subsubsection{Physiological Measures}

Heart rate (HR) was measured using a Polar heart rate monitor (Polar Electro, Finland). Body core temperature $\left(T_{c}\right)$ was measured using a pill size indigestible thermistor and a radio receiver (HQ Inc., Florida). This device provides a core temperature measurement that is intermediate between rectal and esophageal temperature. ${ }^{60}$ Subjects ingested the capsule approximately 12 hours before undertaking the protocol to ensure proper location of the capsule within the 
intestinal tract. Physiological strain was determined by: PhSI $=5 *\left[\left(\mathrm{~T}_{\mathrm{c} \cdot \mathrm{t}}-\mathrm{T}_{\mathrm{c} \cdot 0}\right) /\left(39.5-\mathrm{T}_{\mathrm{c} \cdot 0)}\right)\right]+$ $5 *\left[\left(\mathrm{HR}_{\mathrm{t}}-\mathrm{HR}_{0}\right) /\left(\mathrm{HR}_{\max }-\mathrm{HR}_{0}\right)\right]$; where $\mathrm{t}=$ time of measurement $(\mathrm{min}), 0=$ baseline measurement, $\mathrm{T}_{\mathrm{c}}=$ body core temperature $\left({ }^{\circ} \mathrm{C}\right)$, and $\mathrm{HR}=$ heart rate $\left(\right.$ beats $\left.\cdot \mathrm{min}^{-1}\right) .{ }^{50}$

\subsubsection{Perceptual Measures}

Rating of perceived exertion and RTS were measured using previously validated scales. Rating of perceived exertion was obtained with the Adult OMNI Walk/Run Perceived Exertion scale (Appendix C). This category metric contains a rating range from 0 , "extremely easy," to 10 "extremely hard". ${ }^{73}$ Thermal sensation was obtained with the novel OMNI Thermal Sensation

Scale (Appendix C) which contains verbal descriptors from the Gagge thermal sensation scale ${ }^{20}$ and numerical categories ranging from 1, "comfortable," to 5 "very hot". Perceptual strain was determined by the equation: PeSI $=5 *\left[\left(\mathrm{TS}_{\mathrm{t}}-1\right) / 4\right]+5 *\left(\mathrm{PE}_{\mathrm{t}} / 10\right)$; where TS $=$ thermal sensation, and $\mathrm{PE}=$ perceived exertion..$^{70}$ The perceptual strain index used in the investigation will be modified from the above original equation by Tikuisis et al. ${ }^{70}$ This modification will incorporate the different perceptual metrics used where PE is perceived exertion as measured by the OMNI RPE scale and TS is thermal sensation as measured by the OMNI RTS scale. 


\subsection{DATA ANALYSIS}

Physiological and perceptual responses were measured at baseline, 20-minutes of the treadmill protocol and at termination of the full 50-minute protocol. Descriptive values of the combined data for anthropometric, physiological, and perceptual variables were calculated as mean \pm standard deviation (SD) and presented for the separate investigations and the combined data set. These responses were statistically examined using a two factor analysis of variance [ANOVA; Investigation $\mathrm{x}$ Time] to separately identify homogeneity between the four interrelated investigations. Data from FIRE I and FIRE II were the mean response of each subject's trials for a given protocol. Separate analyses were conducted for each physiological and perceptual response. Statistical significance was accepted at the $\mathrm{p}<0.05$ level.

Regression analyses between body core temperature and both RPE and RTS were performed separately for data obtained from each of the four interrelated investigations. In addition, responses from all subjects in the four interrelated investigations were combined into a single dataset. These combined data were used to construct the PHI. Regression analyses examined the relation between body core temperature and the perceptual responses. This regression analysis provided the level of relation between RPE and RTS for a measured body core temperature.

Using a modification of the concept proposed by Moran et al..$^{50}$, a graphical presentation consisting of RPE on the horizontal axis and RTS on the vertical axis was constructed. As employed by Moran et al. ${ }^{50}$ colored zones representing the different levels of exertional heat strain were determined within the ranges of RPE, RTS and $T_{c}$ recorded across the four

investigations examined presently. Moran et al. ${ }^{50}$ used rectal temperature as a measure of body core temperature. Each colored zone of the PHI will encompass several levels of exertional heat 
strain similar to that presented by Moran et al. ${ }^{50}$ and provided a specific range of $\mathrm{T}_{\mathrm{c}}$ (Table 4).

The body core temperature ranges for each PHI zone used data from the present investigation.

Table 4. Levels of exertional heat strain associated with the calculated PSI and the corresponding measured $T_{\text {re }}$ matched with the colored zones for the newly developed PHI.

\begin{tabular}{|c|c|c|c|}
\hline Strain & PSI & $\mathrm{T}_{\mathrm{re}}\left({ }^{\circ} \mathrm{C}\right)$ & PHI Zone \\
\hline \multirow{3}{*}{ No/Little } & 0 & $37.12 \pm 0.03$ & \multirow{3}{*}{ Green } \\
\hline & 1 & $37.15 \pm 0.04$ & \\
\hline & 2 & $37.35 \pm 0.03$ & \\
\hline \multirow[t]{2}{*}{ Low } & 3 & $37.61 \pm 0.03$ & \multirow{3}{*}{ Yellow } \\
\hline & 4 & $37.77 \pm 0.04$ & \\
\hline \multirow{2}{*}{ Moderate } & 5 & $37.99 \pm 0.05$ & \\
\hline & 6 & $38.27 \pm 0.07$ & \multirow{4}{*}{ Red } \\
\hline High & 7 & $38.60 \pm 0.04$ & \\
\hline & 8 & 38.70 & \\
\hline Very High & $\begin{array}{c}9 \\
10\end{array}$ & No data available & \\
\hline
\end{tabular}

*Values are means \pm SE from Moran, D. S., Shitzer, A., \& Pandolf, K. B. (1998). A Physiological Strain Index to Evaluate Heat Stress. Am J Physiol. Regul Integr Comp Physiol , 275, R129-R134.

In order to include the combination of RPE and RTS for the full range of physiologically sustainable $T_{c}$ prior to cell-death (i.e. the full $T_{c}$ range commensurate with cell life) it was necessary to estimate certain sectors of the newly developed PHI. The PeSI was used for this purpose by mathematically determining the level of risk for each PHI sector that require responses that exceed the measurement limits of the protocols employed presently (Figure 2; high RPE/low RTS and low RPE/high RTS). This was done by solving for RPE at a given RTS; and solving for RTS at a given RPE using estimated PeSI values. 


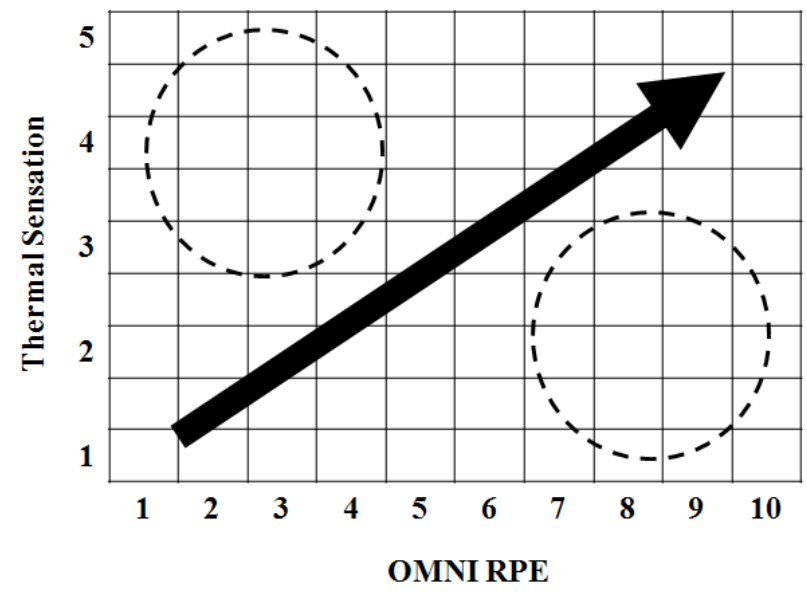

Figure 2. A Hypothetical PHI. Areas of high RPE with low RTS and low RPE with high RTS (hyphenated circles) that are mathematically estimated using PeSI. The solid arrow represents the increase in body core temperature with increases in RPE and RTS.

It was assumed that there is a similar physiological response between the measured sectors of the PHI and the estimated sectors of the PHI. An example of this concept is a measured RPE of 5 and RTS of 4 when entered into the PeSI would result in a calculated value of 6.25 . The body core temperature measured concurrently with these ratings is $38.0^{\circ} \mathrm{C}$ with a range of 37.6 to $38.4^{\circ} \mathrm{C}$. Therefore, a PeSI of 6.25 would be linked to a body core temperature of $38.0^{\circ} \mathrm{C}$ with a range of 37.6 to $38.4^{\circ} \mathrm{C}$. Using the estimation procedure, an estimated RPE of 10 and RTS of 2, which exceed the measurement limits of the protocols employed presently, when entered into the PeSI would also result in a calculated value of 6.25. It was assumed then that a RPE of 10 and RTS of 2 would produce a similar physiological response as a RPE of 5 and RTS of 4 . In this case, both sets of responses would be linked to a mean body core temperature of $38.0^{\circ} \mathrm{C}$ with a range of 37.6 to $38.4^{\circ} \mathrm{C}$. 


\subsection{RESULTS}

This investigation developed a perceptual hyperthermia index to evaluate exertional heat strain during treadmill exercise in firefighters and young adults who did not have firefighting experience. The investigation employed a combined data set from four interrelated investigations involving exercise induced thermal stress.

\subsection{DESCRIPTIVE INFORMATION}

The means $( \pm \mathrm{SD})$ of the physiological and perceptual responses for each measurement point are presented separately for the four interrelated investigations and for the combined data set (Table 5).

Table 5. Physiological and perceptual responses during treadmill exercise in the heat. Mean \pm SD

\begin{tabular}{llccccc}
\hline & Combined & FIRE I & FIRE II & COG I & COG II \\
\hline $\mathbf{T}_{\mathbf{c}}$ & Baseline & $37.04 \pm 0.35$ & $37.09 \pm 0.52$ & $36.96 \pm 0.20$ & $36.91 \pm 0.28$ & $37.14 \pm 0.30$ \\
$\left({ }^{\circ} \mathbf{C}\right)$ & 20-min & $37.60 \pm 0.30$ & $37.76 \pm 0.26$ & $37.52 \pm 0.20$ & $37.54 \pm 0.42$ & $37.60 \pm 0.31$ \\
& Termination & $38.53 \pm 0.58$ & $38.21 \pm 0.58$ & $38.24 \pm 0.28$ & $39.03 \pm 0.48$ & $38.85 \pm 0.51$ \\
\hline $\mathbf{H R}$ & Baseline & $90.54 \pm 16.78$ & $98.82 \pm 11.5$ & $95.19 \pm 10.54$ & $63.00 \pm 10.7$ & $92.79 \pm 13.9$ \\
$\left(\mathbf{b} \cdot \mathbf{m i n}^{-1}\right)$ & 20-min & $147.26 \pm 21.60$ & $170.30 \pm 11.9$ & $148.64 \pm 19.42$ & $129.9 \pm 18.8$ & $141.74 \pm 18.5$ \\
& Termination & $170.29 \pm 16.75$ & $178.52 \pm 7.50$ & $166.69 \pm 16.38$ & $166.6 \pm 20.4$ & $166.89 \pm 18.9$ \\
\hline $\mathbf{R P E}$ & Baseline & $0.24 \pm 0.42$ & $0.15 \pm 0.31$ & $0.15 \pm 0.20$ & $0.30 \pm 0.48$ & $0.37 \pm 0.60$ \\
$(\mathbf{0 - 1 0})$ & 20-min & $4.88 \pm 1.63$ & $6.52 \pm 1.55$ & $4.75 \pm 1.50$ & $3.70 \pm 0.95$ & $4.68 \pm 1.42$ \\
& Termination & $7.43 \pm 1.86$ & $7.54 \pm 1.92$ & $6.96 \pm 2.07$ & $7.30 \pm 1.83$ & $7.84 \pm 1.64$ \\
\hline $\mathbf{R T S}$ & Baseline & $1.78 \pm 0.77$ & $1.65 \pm 0.68$ & $1.46 \pm 0.59$ & $1.60 \pm 0.70$ & $2.32 \pm 0.82$ \\
$(\mathbf{1 - 5 )}$ & 20-min & $3.65 \pm 0.67$ & $4.03 \pm 0.52$ & $3.68 \pm 0.64$ & $3.10 \pm 0.57$ & $3.68 \pm 0.67$ \\
& Termination & $4.50 \pm 0.68$ & $4.28 \pm 0.74$ & $4.58 \pm 0.68$ & $4.50 \pm 0.71$ & $4.63 \pm 0.60$ \\
\hline Where $\mathbf{T}_{\mathbf{c}}$ is body core temperature, HR is heart rate, RPE is rating of perceived exertion, and RTS is \\
thermal sensation.
\end{tabular}




\subsubsection{Analysis of Homogeneity}

Homogeneity between the four interrelated investigations was examined with a two-factor analysis of variance [ANOVA; Investigation $\mathrm{x}$ Time] calculated separately for each physiological and perceptual variable.

\subsubsection{Body Core Temperature}

The ANOVA of the body core temperature responses indicated that the investigation $\mathrm{x}$ time interaction $\left(F_{6,176}=6.620, p<0.001, \eta^{2}=0.184\right)$ was significant. Additionally, the investigation main effect $\left(F_{3,176}=6.096, p=0.001, \eta^{2}=0.094\right)$ and the time main effect $\left(F_{2,176}=254.659, p<\right.$ $\left.0.001, \eta^{2}=0.743\right)$ were significant.

Post-hoc analyses of the interaction examined the simple effect of investigation at each time point. There were no significant simple effects of investigation at baseline $\left(F_{1,176}=0.002, p\right.$ $\left.=0.965, \eta^{2}<0.001\right)$ and at $20-\operatorname{mins}\left(F_{1,176}=0.496, p=0.482, \eta^{2}=0.003\right)$. There was a significant simple effect of investigation at termination $\left(F_{1,176}=52.134, p<0.001, \eta^{2}=0.229\right)$. Simple comparisons found that termination body core temperature in FIRE I was significantly lower than termination body core temperature in COG I $\left(F_{1,176}=29.051, p<0.001, \eta^{2}=0.142\right)$ and in COG II $\left(F_{1,176}=25.480, p<0.001, \eta^{2}=0.126\right)$. Simple comparisons found that termination body core temperature in FIRE II was significantly lower than termination body core temperature in COG I $\left(F_{1,176}=26.957, p<0.001, \eta^{2}=0.133\right)$ and in COG II $\left(F_{1,176}=23.141, p<\right.$ $0.001, \eta^{2}=0.116$ ). Termination body core temperature was not significantly different between FIRE I and FIRE II $\left(F_{1,176}=0.002, p=0.965, \eta^{2}<0.001\right)$ and between COG I and COG II $\left(F_{1,176}\right.$ $\left.=1.420, p=0.235, \eta^{2}=0.008\right)$. 


\subsubsection{Heart Rate}

The ANOVA of the heart rate responses indicated that the investigation $\mathrm{x}$ time interaction $\left(F_{6,176}\right.$ $\left.=4.045, p=0.001, \eta^{2}=0.121\right)$ was significant. Additionally, the investigation main effect $\left(F_{3,176}\right.$ $\left.=22.967, p=0.001, \eta^{2}=0.281\right)$ and the time main effect $\left(F_{2,176}=463.736, p<0.001, \eta^{2}=\right.$ $0.121)$ were significant.

Post-hoc analyses of the interaction examined the simple effect of investigation at each time point. There was no significant simple effect of investigation at termination $\left(F_{1,176}=2.588\right.$, $\left.p=0.109, \eta^{2}=0.014\right)$. However, there were significant simple effects at baseline $\left(F_{1,176}=\right.$ 23.383, $\left.p<0.001, \eta^{2}=0.117\right)$ and at $20-\operatorname{mins}\left(F_{1,176}=31.588, p<0.001, \eta^{2}=0.152\right)$.

Simple comparisons examined differences between investigations at baseline and 20mins. Baseline heart rate in COG I was significantly lower than baseline heart rate in FIRE I $\left(F_{1,176}=34.816, p<0.001, \eta^{2}=0.165\right)$, in FIRE II $\left(F_{1,176}=28.130, p<0.001, \eta^{2}=0.138\right)$, and in COG II $\left(F_{1,176}=24.547, p<0.001, \eta^{2}=0.122\right)$. Baseline heart rate was not significantly different between FIRE I and FIRE II $\left(F_{1,176}=0.499, p=0.481, \eta^{2}=0.003\right)$, FIRE I and COG II $\left(F_{1,176}=1.417, p=0.235, \eta^{2}=0.008\right)$, and FIRE II and COG II $\left(F_{1,176}=0.226, p=0.635, \eta^{2}=\right.$ $0.001)$.

Heart rate after 20-mins exercise in FIRE I was significantly higher than heart rate after 20 -mins exercise in FIRE II $\left(F_{1,176}=13.528, p<0.001, \eta^{2}=0.071\right)$, in COG I $\left(F_{1,176}=36.101, p\right.$ $\left.<0.001, \eta^{2}=0.170\right)$, and in COG II $\left(F_{1,176}=24.002, p<0.001, \eta^{2}=0.120\right)$. Heart rate after 20mins exercise in FIRE II was significantly higher than in COG I $\left(F_{1,176}=9.531, p=0.002, \eta^{2}=\right.$ 0.051). Heart rate after 20-mins exercise was not significantly different between FIRE II and $\operatorname{COG}$ II $\left(F_{1,176}=1.859, p=0.174, \eta^{2}=0.010\right)$ and between COG I and COG II $\left(F_{1,176}=3.876, p\right.$ $=0.051, \eta^{2}=0.022$ ). 


\subsubsection{Ratings of Perceived Exertion}

The ANOVA of the perceived exertion responses indicated that the investigation $\mathrm{x}$ time interaction $\left(F_{6,176}=3.237, p=0.005, \eta^{2}=0.099\right)$ was significant. Additionally, the investigation main effect $\left(F_{3,176}=3.915, p=0.010, \eta^{2}=0.063\right)$ and the time main effect $\left(F_{2,176}=425.783, p<\right.$ $\left.0.001, \eta^{2}=0.829\right)$ were significant.

Post-hoc analyses of the interaction examined the simple effect of investigation at each time point. There were no significant simple effects of investigation at baseline $\left(F_{1,176}=0.278, p\right.$ $\left.=0.598, \eta^{2}=0.002\right)$ and at termination $\left(F_{1,176}=0.826, p=0.365, \eta^{2}=0.005\right)$. There was a significant simple effect of investigation at 20-mins $\left(F_{1,176}=14.753, p<0.001, \eta^{2}=0.077\right)$.

Simple comparisons examined differences between investigations at 20-mins. Perceived exertion after 20-mins exercise in FIRE I was significantly higher than perceived exertion after 20 -mins exercise in FIRE II $\left(F_{1,176}=11.316, p<0.001, \eta^{2}=0.060\right)$, in COG I $\left(F_{1,176}=22.071, p\right.$ $\left.<0.001, \eta^{2}=0.111\right)$, and in COG II $\left(F_{1,176}=12.423, p<0.001, \eta^{2}=0.066\right)$. Perceived exertion after 20-mins exercise was not significantly different between FIRE II and COG I $\left(F_{1,176}=3.765\right.$, $\left.p=0.054, \eta^{2}=0.021\right)$, FIRE II and COG II $\left(F_{1,176}=0.021, p=0.884, \eta^{2}<0.001\right)$ and COG I and COG II $\left(F_{1,176}=3.371, p=0.068, \eta^{2}=0.019\right)$.

\subsubsection{Ratings of Thermal Sensation}

The ANOVA of the thermal sensation responses indicated that the investigation $\mathrm{x}$ time interaction $\left(F_{6,176}=3.186, p<0.001, \eta^{2}=0.098\right)$ was significant. Additionally, the investigation main effect $\left(F_{3,176}=3.810, p=0.011, \eta^{2}=0.061\right)$ and the time main effect $\left(F_{2,176}=282.886, p<\right.$ $\left.0.001, \eta^{2}=0.749\right)$ were significant.

Post-hoc analyses of the interaction examined the simple effect of investigation at each time point. There was no significant simple effect of investigation at termination $\left(F_{1,176}=0.649\right.$, 
$\left.p=0.422, \eta^{2}=0.004\right)$. However, there were significant simple effects at baseline $\left(F_{1,176}=5.500\right.$, $\left.p=0.020, \eta^{2}=0.030\right)$ and at $20-\operatorname{mins}\left(F_{1,176}=6.302, p=0.013, \eta^{2}=0.035\right)$.

Simple comparisons examined differences between investigations at baseline and 20mins. Baseline thermal sensation in COG II was significantly higher than baseline thermal sensation in FIRE I $\left(F_{1,176}=9.182, p=0.003, \eta^{2}=0.050\right)$, in FIRE II $\left(F_{1,176}=15.000, p<0.001\right.$, $\left.\eta^{2}=0.079\right)$, and in COG I $\left(F_{1,176}=7.448, p=0.007, \eta^{2}=0.041\right)$. There were no significant differences between FIRE I and FIRE II $\left(F_{1,176}=0.692, p=0.407, \eta^{2}=0.004\right)$, FIRE I and COG I $\left(F_{1,176}=0.031, p=0.860, \eta^{2}<0.001\right)$, and FIRE II and COG I $\left(F_{1,176}=0.277, p=0.599, \eta^{2}=\right.$ $0.002)$.

Thermal sensation after 20-mins exercise in COG I was significantly lower than thermal sensation after 20-mins exercise in FIRE I $\left(F_{1,176}=10.071, p=0.002, \eta^{2}=0.054\right)$, in FIRE II $\left(F_{1,176}=4.716, p=0.031, \eta^{2}=0.026\right)$, and in COG II $\left(F_{1,176}=4.961, p=0.027, \eta^{2}=0.027\right)$. Thermal sensation after 20-mins exercise was not significantly different between FIRE I and FIRE II $\left(F_{1,176}=1.919, p=0.168, \eta^{2}=0.011\right)$, FIRE I and COG II $\left(F_{1,176}=1.858, p=0.175, \eta^{2}\right.$ $=0.010)$, and FIRE II and COG II $\left(F_{1,176}=0.002, p=0.967, \eta^{2}<0.001\right)$.

\subsubsection{Relation between Physiological and Perceptual Responses}

Regression analyses examined the relation between physiological and perceptual responses across measurement time points separately for each of the four interrelated investigations and for the combined data set (Table 6). 
Table 6. Correlation coefficients between body core temperature $\left(T_{c}\right)$, ratings of perceived exertion (RPE), and ratings of thermal sensation (RTS).

\begin{tabular}{|c|c|c|c|c|c|}
\hline Variables & Combined & FIRE I & FIRE II & COG I & COG II \\
\hline$\overline{T_{c}-R P E}$ & $\overline{0.749 *}$ & $\overline{0.742^{*}}$ & $\overline{0.759^{*}}$ & $\overline{0.856^{*}}$ & $\overline{0.766^{*}}$ \\
\hline$T_{c}-$ RTS & $0.713^{*}$ & $0.679 *$ & $0.789 *$ & $0.826^{*}$ & $0.688^{*}$ \\
\hline RPE - RTS & $0.881 *$ & $0.931 *$ & $0.916^{*}$ & $0.883^{*}$ & $0.822 *$ \\
\hline RPE - HR & $0.866^{*}$ & $0.916^{*}$ & $0.878 *$ & $0.877^{*}$ & $0.862 *$ \\
\hline PeSI - PhSI & $0.893 *$ & $0.940 *$ & $0.904 *$ & $0.926^{*}$ & $0.862 *$ \\
\hline
\end{tabular}

Moderate-to-strong correlations were found between body core temperature and perceived exertion ( $\mathrm{r}=0.742$ to 0.856 ). Similarly, moderate-to-strong correlations were found between body core temperature and thermal sensation $(r=0.679$ to 0.826$)$. Strong correlations were found between perceived exertion and thermal sensation $(r=0.822$ to 0.936$)$ and between perceived exertion and heart rate $(r=0.862$ to $r=0.916)$. These findings held for the four interrelated investigations and the combined data set.

\subsection{HEAT STRAIN INDICES}

Physiological (PhSI) and perceptual (PeSI) heat strain indices were calculated for the combined data set. These indices assessed the level of heat strain experienced by the subjects as they performed treadmill exercise while wearing thermal protective clothing.

\subsubsection{Physiological Heat Strain Index}

The calculated PhSI rating increased from $0.00( \pm 0.00)$ at baseline to $4.71( \pm 1.16)$ after 20 -mins, and reached $8.00( \pm 1.23)$ at termination of exercise. The PhSI rating was significantly correlated $(\mathrm{p}<0.01)$ with body core temperature $(r=0.877)$ and heart rate $(r=0.876)$. Heat strain zones 
were formed to encompass a range of responses such that a rating of 0.01 to 1.00 was zone 1 , 1.01 to 2.00 was zone 2, extending up to a rating of 9.01 to 10.00 which was zone 10 . Mean $( \pm \mathrm{SD})$ of body core temperatures from the present investigation are presented for each $\mathrm{PhSI}$ rating from 0 to 10 (Table 7). Three data points appear for each subject in the $\mathrm{PhSI}$ ratings representing baseline, 20-mins, and termination. The unequal number in the frequency tabulation for a given PhSI occurred because not every individual rated perceived exertion and thermal sensation the same for similar body core temperatures.

Table 7. Comparison of body core temperature between those reported by Moran et al. and those measured in the present investigation. Mean \pm SD

\begin{tabular}{cccc}
\hline PhSI & $\begin{array}{c}\text { Moran } \\
\mathbf{T}_{\mathbf{r e}}\left({ }^{\circ} \mathbf{C}\right)\end{array}$ & $\begin{array}{c}\text { Gallagher } \\
\mathbf{T}_{\mathbf{c}}\left({ }^{\circ} \mathbf{C}\right)\end{array}$ & $\begin{array}{c}\text { Response } \\
\text { Frequency }\end{array}$ \\
\hline 0 & $37.12 \pm 0.03$ & $37.04 \pm 0.35$ & 65 \\
1 & $37.15 \pm 0.04$ & -- & 0 \\
2 & $37.35 \pm 0.03$ & -- & 0 \\
3 & $37.61 \pm 0.03$ & $37.31 \pm 0.26$ & 5 \\
4 & $37.77 \pm 0.04$ & $37.48 \pm 0.26$ & 13 \\
5 & $37.99 \pm 0.05$ & $37.49 \pm 0.20$ & 17 \\
6 & $38.27 \pm 0.07$ & $37.81 \pm 0.27$ & 21 \\
7 & $38.60 \pm 0.04$ & $37.78 \pm 0.31$ & 14 \\
8 & $38.70 \dagger$ & $38.21 \pm 0.16$ & 21 \\
9 & No data available & $38.81 \pm 0.17$ & 20 \\
10 & & $39.39 \pm 0.15$ & 12 \\
\hline $\mathrm{T}_{\mathrm{re}}:$ rectal & temperature, $\mathrm{T}_{\mathbf{c}}:$ core temperature using ingestible
\end{tabular}

thermometric pill, Response Frequency: number of occurrences for a given PhSI. $\dagger$ indicates only one subject achieved the given PhSI in the study by Moran et al.

\subsubsection{Perceptual Heat Strain Index}

The calculated PeSI rating increased from $1.10( \pm 1.01)$ at baseline to $5.75( \pm 1.51)$ after 20-mins, and reached $8.09( \pm 1.59)$ at termination of exercise. The PeSI was significantly correlated $(\mathrm{p}<$ $0.01)$ with RPE $(r=0.969)$ and RTS $(r=0.970)$. Exertional heat strain zones were formed to encompass a range of responses such that a rating of 0.01 to 1.00 was zone $1,1.01$ to 2.00 was 
zone 2, extending up to a rating of 9.01 to 10.00 which was zone 10 . Mean $( \pm \mathrm{SD})$ of body core temperatures from the present investigation are presented for each PeSI rating from 0 to 10 (Table 8).

Table 8. Body core temperatures

$\left(T_{c}\right)$ for each calculated perceptual

strain rating. Mean $\pm S D$.

\begin{tabular}{ccc}
\hline PeSI & $\mathbf{T}_{\mathbf{c}}\left({ }^{\circ} \mathbf{C}\right)$ & Frequency \\
\hline 0 & $37.08 \pm 0.38$ & 13 \\
1 & $36.96 \pm 0.45$ & 19 \\
2 & $37.06 \pm 0.29$ & 22 \\
3 & $37.16 \pm 0.29$ & 10 \\
4 & $37.43 \pm 0.28$ & 11 \\
5 & $37.71 \pm 0.61$ & 21 \\
6 & $38.02 \pm 0.45$ & 8 \\
7 & $37.91 \pm 0.50$ & 22 \\
8 & $38.00 \pm 0.64$ & 19 \\
9 & $38.58 \pm 0.61$ & 25 \\
10 & $38.54 \pm 0.57$ & 18 \\
\hline Frequency & represents the frequency of \\
occurrence of the calculated PeSI.
\end{tabular}

\subsection{DEVELOPMENT OF THE PHI}

Figure 3 presents PeSI ratings for a concurrent rating of perceived exertion and thermal sensation. These ratings were used as the initial step in the development of the PHI. Due to the use of mean responses in FIRE I and FIRE II, some PeSI ratings were not explicitly presented and were rounded up to the next PeSI rating (i.e. 6.33 becomes 6.5). 


\begin{tabular}{|c|c|c|c|c|c|c|c|c|c|c|c|}
\hline 55 & 5.0 & 5.5 & 6.0 & 6.5 & 7.0 & 7.5 & 8.0 & 8.5 & 9.0 & 9.5 & 10.0 \\
\hline & 3.75 & 4.25 & 4.75 & 5.25 & 5.75 & 6.25 & 6.75 & 7.25 & 7.75 & 8.25 & 8.75 \\
\hline & 2.5 & 3.0 & 3.5 & 4.0 & 4.5 & 5.0 & 5.5 & 6.0 & 6.5 & 7.0 & 7.5 \\
\hline 2 & 1.25 & 1.75 & 2.25 & 2.75 & 3.25 & 3.75 & 4.25 & 4.75 & 5.25 & 5.75 & 6.25 \\
\hline 1 & 0 & 0.5 & 1.0 & 1.5 & 2.0 & 2.5 & 3.0 & 3.5 & 4.0 & 4.5 & 5.0 \\
\hline & 0 & 1 & 2 & 3 & 4 & 5 & 6 & 7 & 8 & 9 & 10 \\
\hline
\end{tabular}

Figure 3. First stage development of the PHI with perceptual strain index rating presented for concurrent rating of perceived exertion and thermal sensation. Values in each cell are perceptual strain index ratings.

Figure 4 presents the mean body core temperatures corresponding to RPE and RTS ratings that equate to a specified PeSI rating. These were reclassified to account for the decimal equivalents of the PeSI ratings not listed in Table 8. Such as 0.1 to 1.0 (PeSI 1) zone which was split into 0.1 to $0.5(0.5)$ and 0.51 to $1.0(1.0)$ zones.

\begin{tabular}{|c|c|c|c|c|c|c|c|c|c|c|c|}
\hline & 37.92 & 37.93 & 38.32 & 37.85 & 38.19 & 38.13 & 37.89 & 38.73 & 38.62 & 38.13 & 38.89 \\
\hline & 37.31 & 37.64 & 37.67 & 37.55 & 38.21 & 37.97 & 37.83 & 37.78 & 38.47 & 38.57 & 38.22 \\
\hline & 37.04 & 37.12 & 37.25 & 37.67 & 37.41 & 37.92 & 37.93 & 38.32 & 37.85 & 38.19 & 38.13 \\
\hline & 37.10 & 37.03 & 37.15 & 37.55 & -- & 37.31 & 37.64 & 37.67 & 37.55 & 38.21 & 37.97 \\
\hline 1 & 37.08 & 36.95 & 36.97 & 37.12 & 36.80 & 37.04 & 37.12 & 37.25 & 37.67 & 37.41 & 37.92 \\
\hline & 0 & 1 & 2 & 3 & 4 & 5 & 6 & 7 & 8 & 9 & 10 \\
\hline
\end{tabular}

Figure 4. Second stage development of the PHI. PeSI ratings were replaced with the concurrent mean body core temperatures. Mean body core temperatures presented in each cell were identified by measured ratings of perceived exertion and thermal sensation. 
The clinical definition of heat exhaustion states that it occurs at a body core temperature between 38.5 and $40.0^{\circ} \mathrm{C}$ and that heat stroke occurs at a body core temperature greater than $40.5^{\circ} \mathrm{C}$. Therefore, it was determined that the upper PHI zone should be colored red as the body core temperature corresponding to the respective RPE-RTS coordinates are consistent with clinical definitions of major heat illness. The lower PHI zone, colored green, includes the normal body core temperature of approximately $37^{\circ} \mathrm{C}$ (range of $36.2^{\circ}$ to $37.7^{\circ} \mathrm{C}$ ). The PHI zone consisting of body core temperatures between the lower and upper PHI zones is colored yellow indicating cautionary physiologic thermal conditions (Table 9).

Table 9. Mean body core temperature ranges for the Perceptual Hyperthermia Index (PHI).

\begin{tabular}{llr}
\hline PHI Ratings & PHI Zone & Range of $\mathbf{T}_{\mathbf{c}}\left({ }^{\circ} \mathbf{C}\right)$ \\
\hline 0 to 4 & Green & $>36.00$ to 37.49 \\
5 to 8 & Yellow & 37.50 to 37.99 \\
9 to 10 & Red & 38.00 to $>40.50$ \\
\hline
\end{tabular}

These mean body core temperatures were then transposed on to the basic conceptual framework for the PHI. A few PeSI ratings produced incongruent mean body core temperatures as indicated by a star in the box (Figure 5). Incongruent mean body core temperatures are temperatures that do not conform to the PHI zone ranges. The mean body core temperature of $38.32^{\circ} \mathrm{C}$ for a rating of perceived exertion of 2 and a rating of thermal sensation of 5 would technically be classified as part of the red zone. However, that rating is surrounded by mean body core temperatures that are representative of the yellow zone. 


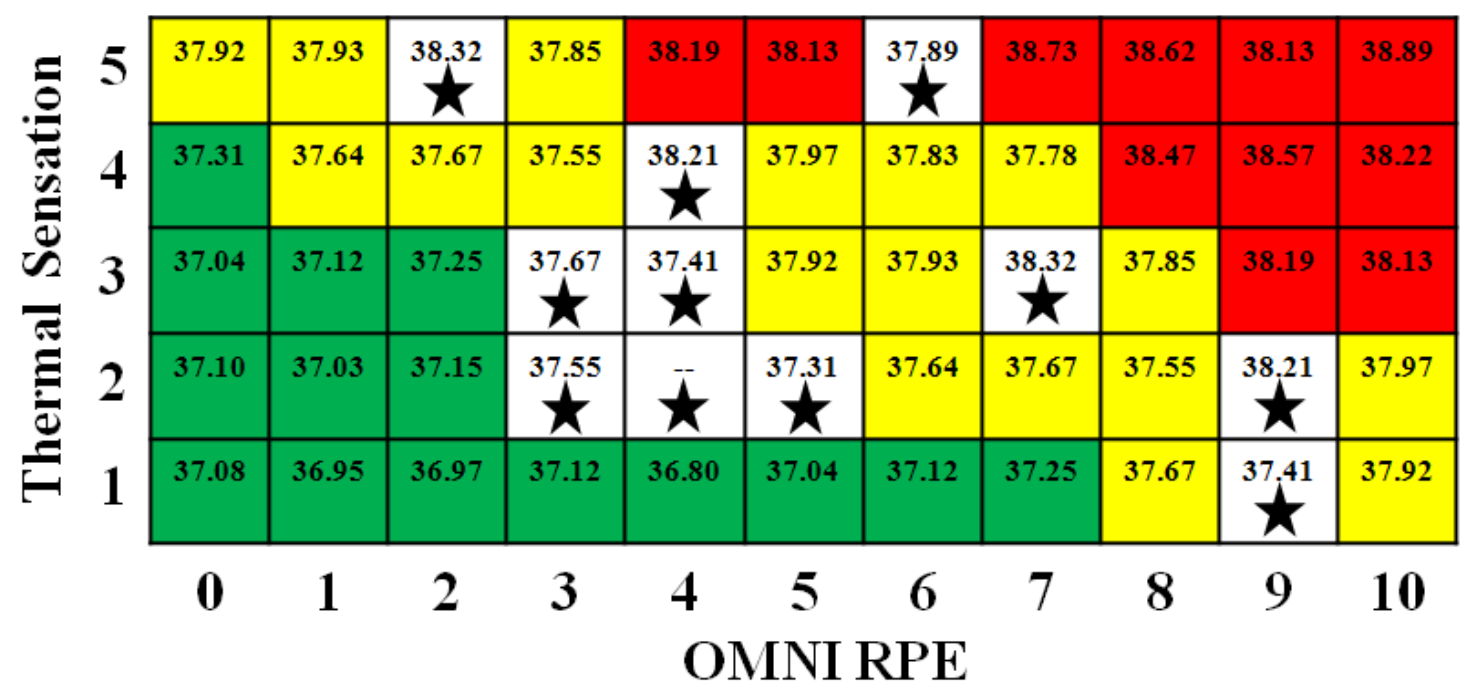

Figure 5. Third stage development superimposes the PHI color scheme over the mean body core temperatures (as presented by each cell) for a given PeSI.

In these circumstances, the zone chosen was based on visually identifying the body core temperature trend. Resolving the zone-color scheme resulted in a completed PHI (Figure 6).

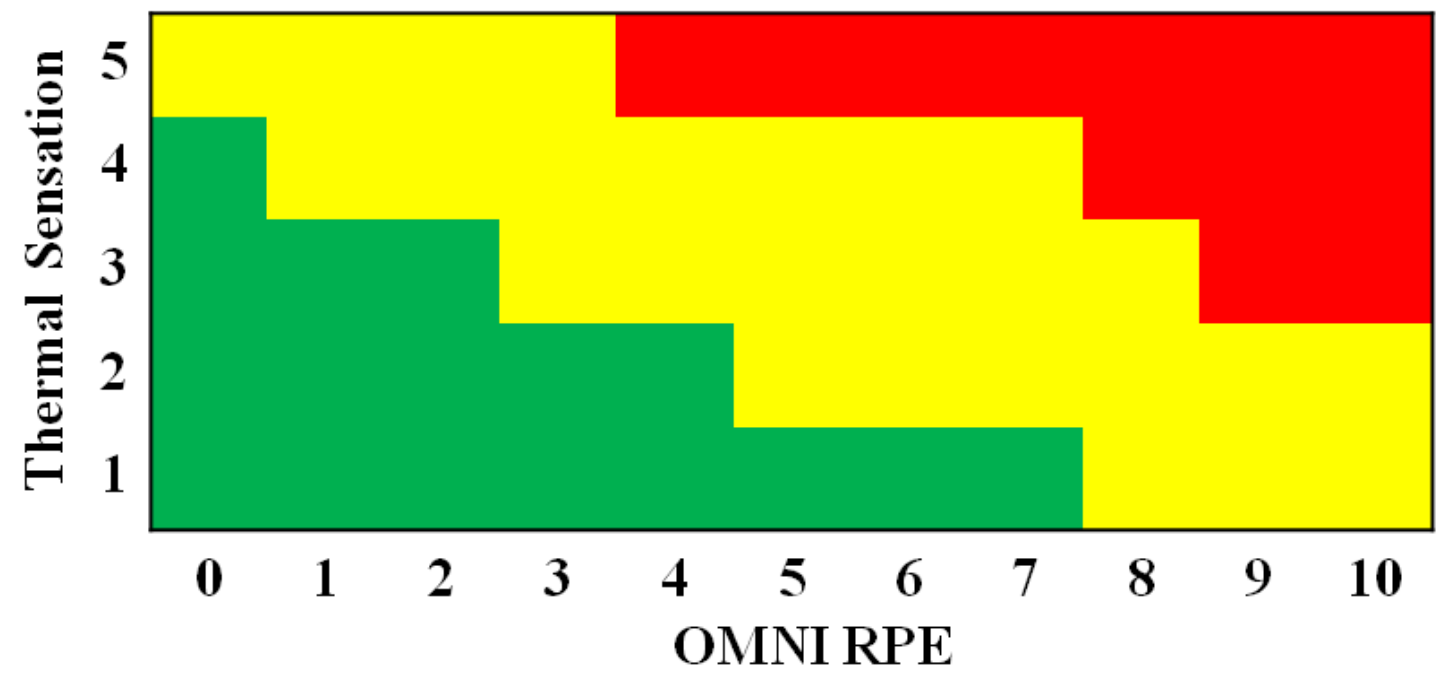

Figure 6. Final Perceptual Hyperthermia Index (PHI). 


\subsection{DISCUSSION, CONCLUSION, AND RECOMMENDATIONS}

This investigation developed the PHI to evaluate exertional heat strain in young adult firefighters and non-firefighters performing treadmill exercise. The PHI used RPE and RTS as surrogate measures of body core temperature response to treadmill exercise with subjects wearing personal protective clothing. The investigation employed an asymmetrical design using the combined data set from four interrelated investigations involving exercise induced thermal stress.

\subsection{HOMOGENEITY OF RESPONSES}

A comparison of the physiological and perceptual responses during treadmill exercise between the four interrelated investigations indicated that they were not homogenous. This was possibly due to the heterogeneous samples across investigations and methodological differences between the four experimental paradigms.

Body core temperature at termination of the experiment was significantly higher in the COG I and II trials as compared to the FIRE I and II trials. COG I and COG II trials were 50-min continuous treadmill exercise and may have elicited a higher metabolic and associated thermal response as compared to the intermittent treadmill exercise protocol employed in the FIRE I and FIRE II trials. 
Baseline heart rate was significantly lower in COG I as compared to the COG II, FIRE I, and FIRE II. It is possible that aerobic fitness influenced the baseline heart rates as the subject sample in COG I had the highest maximum oxygen consumption. However, it should be noted that the difference in aerobic fitness level may also be due to the differences between experiments in the testing protocols to establish maximum or peak oxygen consumption. FIRE I and FIRE II employed a peak cycle ergometer stress test whereas COG I and COG II employed a maximum treadmill stress test.

Heart rate after 20-mins exercise was significantly higher in FIRE I as compared to FIRE II, COG I, and COG II. The higher HR may be due to the protocol requirement that subjects carried a bodybar in FIRE I but not in the other trials. This external weight was intended to incorporate upper body muscle groups into the exercise forcing function.

Similar to the HR response after 20-mins of exercise, RPE was significantly higher in FIRE I as compared to the FIRE II, COG I, and COG II trials. Here again, the higher RPE may have occurred because a bodybar was carried during treadmill exercise in the FIRE I trial but not in the other trials.

Baseline thermal sensation was significantly higher in COG II as compared to COG I, FIRE I, and FIRE II. There are no plausible explanations for this difference as all four interrelated investigations employed the same thermal protective clothing ensemble and exercise was performed in the same heated room environment. It is particularly interesting that COG I and COG II differed in RTS yet they employed identical experimental paradigms.

Thermal sensation, after 20-mins of exercise, was significantly lower in COG I as compared to COG II, FIRE I, and FIRE II. Possible explanations for this difference may be that COG I employed a smaller sample size (ten subjects) as compared to the eighteen or nineteen 
subjects in each of the other three trials. In addition, COG I subjects had a higher maximum oxygen uptake than subjects in the other trials. Given equal ambient thermal conditions, aerobic fitness attenuates thermal strain and by extension thermal sensation.

Although there were significant differences in body core temperature, HR, RPE, and RTS between the four interrelated investigations, these differences may be beneficial and provide a more generalizable scenario in which the newly developed PHI could be used. The present findings suggest that the PHI could be employed during either continuous or intermittent horizontal exercise (treadmill) with the individual holding equipment similar to an ax or ladder (bodybar). The PHI also takes into consideration a range of subject characteristics that may encompass varying levels of firefighting experience from new recruit to novice to experienced firefighter.

\subsection{PHYSIOLOGICAL RESPONSES}

The physiological responses recorded for the four interrelated investigations were similar to those published previously for firefighters performing fire-task simulation ${ }^{29,75}$, during treadmill exercise in personal protective equipment ${ }^{31}$, and in the development of heat strain indices..$^{50,70}$ Von Heimburg ${ }^{75}$ observed heart rate responses of $167 \mathrm{~b} \cdot \mathrm{min}^{-1}$ in rescuers upon reaching the top of the stairs. The same individuals achieved a heart rate of $182 \mathrm{~b} \cdot \mathrm{min}^{-1}$ at the end of the task during simulated rescue of hospital patients which included climbing six floors and rescuing six manikin patients. Similarly, Holmer and Gavhed ${ }^{29}$ showed that heart rates averaged $168 \mathrm{~b} \cdot \mathrm{min}^{-1}$ for a 22-min simulated fire fighting activity and an average heart rate of $179 \mathrm{~b} \cdot \mathrm{min}^{-1}$ for the heaviest of work tasks during the simulation. The mean heart rate achieved at exercise 
termination for the present investigation was $170 \mathrm{~b} \cdot \mathrm{min}^{-1}$. However, the heart rate was 178 $\mathrm{b} \cdot \mathrm{min}^{-1}$ for the subjects in FIRE I who periodically carried a bodybar during the treadmill exercise. This value closely resembled the heart rate observed at the end of the simulated fire task reported by von Heimburg ${ }^{75}$ and the heaviest of works tasks used by Holmer and Gavhed. ${ }^{29}$

Body core temperatures from the present investigation ranged from 37.04 to $38.53^{\circ} \mathrm{C}$ and are similar to those reported by Moran et al. ${ }^{50}$ when developing the physiological heat strain index $\left(37.12\right.$ to $\left.38.7^{\circ} \mathrm{C}\right)$ and by Tikuisis et al. ${ }^{70}$ when developing the perceptual heat strain index $\left(37.1\right.$ to $38.3^{\circ} \mathrm{C}$ ). The four interrelated experimental paradigms employed presently resulted in a range of body core temperatures sufficient for the development of the perceptual hyperthermia index (PHI).

\subsection{PERCEPTUAL RESPONSES}

The perceptual responses recorded during the four interrelated investigations were both similar and different to those reported previously under thermally stressful conditions during cycling and upper body exercise. Galloway and Maughan ${ }^{21}$ recorded Borg Scale RPE during exhaustive cycling exercise at $70 \%$ of $\mathrm{VO}_{2 \text { peak }}$ in a warm $\left(31^{\circ} \mathrm{C}, 70 \%\right.$ relative humidity) environment. BorgRPE was 13 after 20-mins of exercise and 18 after 50-mins of exercise. These Borg-RPEs generally convert to OMNI-RPEs of 5 and 9 , respectively ${ }^{64}$. In the present investigation, OMNI-

RPE was 5 after 20-mins and 7 at exercise termination. Using the inter-scale conversion, the OMNI-RPE from the present investigation at termination was lower than the Borg-RPE reported by Galloway and Maughan ${ }^{21}$ at 50 -mins of exercise. Differences between investigations in the RPE at the end of exercise may be due to differences between cycling in comfortable clothing 
and treadmill walking in thermal protective clothing. However, some of the subjects in the present investigation did not complete a full 50-mins of exercise and therefore, did not reach RPEs at the high end of the scale. In these individuals, elevated levels of thermal sensation may have contributed to shorter durations of exercise. It was expected that treadmill exercise while wearing thermal protective clothing would produce a greater thermal strain on the individuals in the present investigation than those performing cycling exercise as reported previously.

More recently, Crewe et al. ${ }^{16}$ examined the rate of increase in RPE as a prediction of cycle exercise duration to fatigue under different environmental conditions. Three of the five exercise conditions were performed in a hot $\left(35^{\circ} \mathrm{C}, 50 \%\right.$ relative humidity, wind speed at 10 $\mathrm{km} / \mathrm{h}$ ) environment at intensities of $55 \%, 60 \%$, and $65 \%$ of peak power output. Borg-RPE after 20 -mins of exercise was found to be 14 in the $55 \%$ trial, 16 in the $60 \%$ trial, and 18 in the $65 \%$ trial. These RPEs (converted to OMNI: 6, 7, 9) were higher than the rating of 5 after 20-mins of exercise in the present investigation. Borg-RPE at termination for all three hot trials was 19 (OMNI 10) which well exceeds the 7 found in the present investigation. Differences between Crewe et al. ${ }^{16}$ and the present investigation could be attributed to the environmental conditions and modes of exercise. Crewe et al. ${ }^{16}$ used an environmental chamber that allowed convective heat loss by the cyclists. The clothing worn in the present investigation provided no heat loss mechanisms for subjects and may have contributed to the individuals terminating exercise due to thermal stress and not purely a function of exertional intolerance at higher exercise intensity.

Additionally, Price and Campbell ${ }^{63}$ examined thermoregulatory responses during prolonged upper-body exercise in cool and warm conditions. Borg-RPE was approximately 15 after 30-mins during exercise in warm ambient conditions $\left(31.5^{\circ} \mathrm{C}, 48.9 \%\right.$ relative humidity). A Borg-RPE of 15 generally equates to an OMNI-RPE of $7^{64}$. The OMNI-RPE of 5 recorded in the 
present investigation after 20-mins of exercise is lower than the level of perceived exertion for prolonged upper body exercise as reported by Price and Campbell. ${ }^{63}$ Although the perceptual results differ between investigations, it is plausible to consider that the additional weight of the thermal protective clothing and self-contained breathing apparatus would induce greater upper body exercise. Price and Campbel1 ${ }^{63}$ used 60 -mins of arm crank exercise at $60 \% \mathrm{VO}_{2 \text { peak }}$, whereas the present investigation employed treadmill mode, i.e. weight bearing aerobic exercise. Arm crank exercise targets a comparatively smaller muscle mass and may produce higher ratings of perceived exertion than treadmill exercise where comparatively large volume of muscle mass is activated.

\subsection{RELATION BETWEEN PHYSIOLOGICAL AND PERCEPTUAL RESPONSES}

The relation between the physiological and perceptual responses to the same thermal stimuli was the basis for the development of the PHI. Cheung ${ }^{14}$ has proposed that occupational exposure standards should not only use physiological outcomes, but also thermal perceptions reflecting the behavioral and exercise response to heat stress. Previous research has produced conflicting results as to the level and the interpretation of the relation between physiological responses and their perceptual analog during exposure to thermally stressful environments. Hoster et al. ${ }^{31}$ reported PeSI was significantly greater than PhSI after 8-mins, 23-mins, and at termination of treadmill exercise for subjects wearing personal protective equipment. Tikuisis et al. ${ }^{70}$ reported that PeSI was lower than PhSI in a trained cohort of highly fit $\left(\mathrm{VO}_{2 \max } \sim 59 \mathrm{ml} \cdot \mathrm{kg}^{-1} \cdot \mathrm{min}^{-1}\right)$ individuals. However, the PeSI was greater than PhSI for the mid-exercise time points in the untrained cohort which consisted of moderately fit $\left(\mathrm{VO}_{2 \max } \sim 44 \mathrm{ml} \cdot \mathrm{kg}^{-1} \cdot \mathrm{min}^{-1}\right)$ individuals. At 
the first measurement time point (15-mins) and the end measurement time point (60-mins), PeSI did not differ from PhSI. Petruzzello et al. ${ }^{62}$ also reported that PeSI was consistently lower than PhSI during laboratory and field simulations. In the Petruzzello investigation ${ }^{62}$, the relation between the PeSI and PhSI showed moderate correlations of $r=0.70$ for a laboratory protocol that examined short-term (15-min) moderate activity in subjects wearing firefighting clothing and $\mathrm{r}=0.70$ for a field protocol that examined short-term ( 18-min) firefighting drills. The present investigation observed a strong correlation $(r=0.893)$ between PeSI and PhSI that was greater than reported by Petruzzello et al. ${ }^{62}$ Additionally, in the present investigation PhSI and PeSI were similar at baseline and at termination of exercise but PeSI was greater than PhSI after 20-mins of exercise. These results differ slightly from Petruzzello et al. ${ }^{62}$ who found that PeSI was less than PhSI. The present findings are however, consistent with those reported by Tikuisis et al. $^{71}$ for an untrained cohort.

Differences between the present investigation and that of Petruzzello et al. ${ }^{62}$ are primarily methodological. Petruzzello et al. ${ }^{62}$ utilized short-duration activity in both the laboratory and field settings as opposed to the longer duration ( $>20$-mins) employed in the present investigation. Petruzzello et al. ${ }^{62}$ also modified the perceptual heat strain indices for both the laboratory and field studies by altering the baseline to a relative value. In this alteration, the initial rating of the individual represented the baseline value. Conversely, Tikuisis et al. ${ }^{71}$ and the present investigation used the minimum category scale rating as the absolute baseline value. It is possible that the different perceptual metrics may also have influenced the calculations where the present investigation is the first to use OMNI RPE and OMNI RTS as compared to previous investigations that employed the Borg RPE and Gagge RTS. 
Brearley and Finn ${ }^{10}$ examined responses of four motor-sport athletes during V8 Supercar racing in hot ambient conditions for both short and long racing distances. During the short race format, the PhSI was 6.5 and the PeSI was 6.7. During the long race format, the PhSI was 8.0 and the PeSI was 7.3. The PhSI was 7.4 and the PeSI was 7.1 for the data when combined over the short and long race distances. Interpretation of the data reported by Brearley and Finn ${ }^{10}$ is limited. Only means and standard deviations were presented owing to the small sample size. Nevertheless, the authors suggested that the information provided by PhSI and PeSI may not differ when employed under the same thermal conditions. Although only an observation, a possible explanation for the PeSI being less than the PhSI for the long race distance and also for the combined data may be due to the head and torso cooling resulting from the type of driver's ensemble worn during V8 Supercar races in warm to hot conditions. ${ }^{10}$ These findings support the development of the PHI in that thermally induced perceptual responses may be an effective surrogate to physiological measures of exertional heat strain.

\subsection{THE PERCEPTUAL HYPERTHERMIA INDEX}

A perceptual hyperthermia index (PHI) was developed using RPE and RTS as surrogate measures of exertional heat strain. The PHI was developed using data obtained from four interrelated investigations that examined physiological, perceptual, and cognitive responses during treadmill exercise for subjects wearing thermal protective clothing. Moderate-to-strong correlations were observed between the physiological measures (body core temperature, heart rate) and the perceptual measures (perceived exertion, thermal sensation). This relation supports the use of perceptual measures to evaluate exertional heat strain. The use of perceptual measures 
in place of physiological measures may be more convenient in the assessment of hyperthermia for individuals performing work under thermal stress, such as firefighting.

The assessment of heat stress and subsequent heat strain under hot, ambient conditions is difficult outside of a laboratory setting. The current knowledge base regarding temperature regulation under heat stress indices indicates the need to develop user friendly methods for quick and accurate assessments in thermally stressful field conditions. The majority of currently employed methods require expensive equipment, planning, and/or physical interaction between the investigator and the subject. One possible means to assess homeostatic disruption of temperature regulation in firefighters is the PHI. The PHI provides a quick, easy and unobtrusive method to assess exertional heat strain while firefighters are actively engaged in fire suppression and rescue.

A few experimental limitations should be noted. The currently developed PHI used four separate cohorts. These cohorts were comprised of individuals who ranged in firefighting experience and subsequent exposure to the thermal stress induced by fires while wearing thermal protective clothing. The PHI may only be generalizable to individuals having the descriptive characteristics of subjects in the four investigations. The four interrelated investigations also differed slightly in methodology where FIRE I and FIRE II employed intermittent treadmill exercise protocols and COG I and COG II employed continuous treadmill exercise protocols. Furthermore, the development of the PHI was not a primary objective of the four investigations. As such, the exercise paradigm employed may not have been optimal in presenting thermally stressful conditions. The PHI range of perceived exertion and thermal sensation that were measured only provided a linear trend and did not encompass all possible combinations of RPE and RTS. It was assumed that similar physiological responses existed between the measured 
sectors of the PHI and the estimated sectors of the PHI. It is possible that this assumption is not consistent with the actual physiological response associated with high perceived exertion and low thermal sensation, or low perceived exertion and high thermal sensation.

Additionally, the completion of the color scheme of the PHI necessitated visual inspection and interpretation for incongruent mean body core temperatures. One possible explanation for the incongruent mean body core temperatures may be related to the low frequency of perceived exertion and thermal sensation combinations. Another possible explanation may pertain to the mix of subject characteristics that involved both volunteers and firefighters. Responses from volunteers may not be similar to that of firefighters. One last possible explanation may be due to the introduction of an additional load (i.e. carrying a bodybar) that may have increased ratings of perceived exertion without a concomitant increase in thermal sensation.

\subsection{SUMMARY}

In summary, four interrelated investigations were combined into one data set from which the PHI was developed. Moderate-to-strong correlations between the physiological and perceptual measures support the use of RPE and RTS to evaluate exertional heat strain during exercise. The development of the PHI to assess the level of risk for exertional heat strain could provide a valuable field-based metric for firefighters engaged in fire suppression and rescue. This metric may be beneficial in high risk environments that threaten the lives of firefighters. The application of a hyperthermia metric reflects the importance of monitoring the health and wellbeing of firefighters performing physiologically taxing duties associated with fire suppression 
and rescue while wearing protective clothing largely impermeable to ambient gases and particulates.

\subsection{RECOMMENDATIONS}

Recommendations are two-fold for the PHI. The first set of recommendations target limitations in the development of the PHI. The second set of recommendations targets the application and generalizability of the PHI.

\subsubsection{Future Research - Experimental Design}

Three research limitations were encountered in the development of the PHI and should be addressed in subsequent development and cross-validation investigations.

1. The development of the PHI used four interrelated investigations that had minor methodological differences. Future investigations should employ designs that focus on the PHI as a primary variable using a single experimental paradigm.

2. The four interrelated investigations employed an exercise and thermal stress forcing function to induce a hyperthermia response. As such, in the development of the PHI, it was assumed that similar physiological responses existed between the measured sectors of the PHI and the estimated sectors of the PHI. Future investigations should examine the physiological and perceptual responses of nine discernable sectors within the PHI (Figure 7). The present investigation observed an expected dose-response relation between OMNI-RPE and RTS beginning in sector one, increasing to sector five, and ending at sectors eight and nine. Sectors 
should be examined using experimental paradigms that manipulate one perceptual variable while holding the other variable constant. Specifically, the RPE and body core temperature should be examined under thermal-neutral conditions (i.e. as expressed in sectors one, two, and three).

3. One of the four interrelated investigations (FIRE I) incorporated carrying a bodybar during treadmill exercise to simulate transporting firefighting equipment while wearing thermal protective clothing. Future investigations should specifically examine the effect of carrying equipment on perceived exertion and thermal sensation as it pertains to the validity of the PHI.

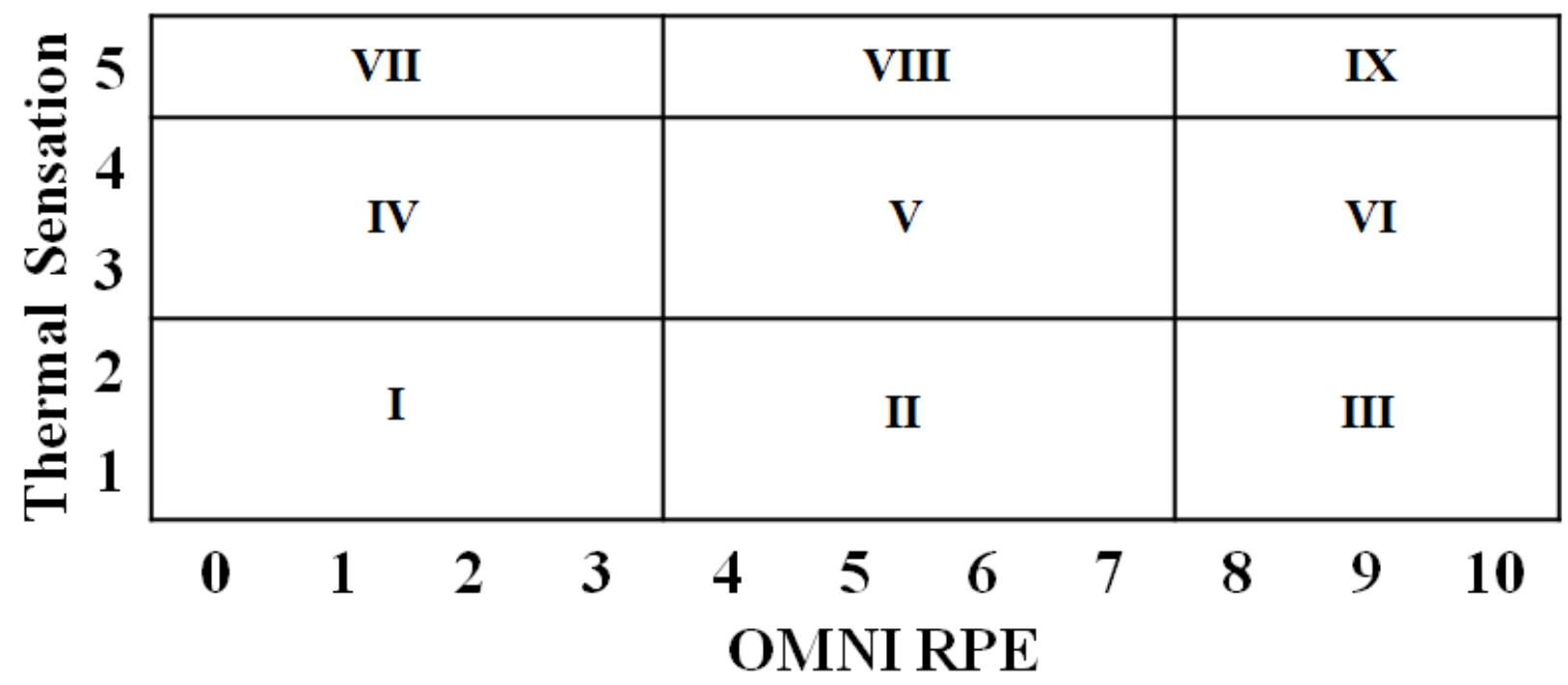

Figure 7. Proposed nine discernable sectors of the PHI to examine separately in future investigations.

\subsubsection{Future Research - PHI Applications}

The applications of the newly developed (prototype) PHI should be examined further in subsequent development and cross-validation investigations. 
1. The sample population employed in the present investigation was comprised of firefighters and also healthy volunteers who had no firefighting experience or experience wearing thermal protective clothing. The influence of firefighting experience on the perceptual responses should be examined further.

2. The sample population employed in the present investigation included a mix of females and males. The influence of gender on physiological and perceptual components of the PHI should be examined further.

3. The present investigation employed four interrelated laboratory investigations. Future investigations should examine the perceptual and physiological responses in field-based settings such as live-fire simulations and firefighting training drills.

4. The present investigation examined physiological and perceptual responses in a heated interior room $\left(98-100^{\circ} \mathrm{F}\right)$. Future investigations should examine the validity of the PHI for use in a larger range of environmental extremes of temperature and humidity.

5. The present investigation examined the physiological and perceptual responses in response to walking on a treadmill at speeds ranging from $2.6-4.5 \mathrm{~km} \cdot \mathrm{hr}^{-1}$. Future investigations should examine the validity of the PHI for use during locomotion speeds and exercise modes unique to firefighting such as climbing, carrying, and crawling.

6. The present investigation developed a perceptual hyperthermia index as a momentary assessment of exertional heat strain during fire suppression and rescue. The educational component associated with the use of the PHI in a field setting should be examined further and may lead to use or modification of the PHI 
during rehabilitation and the decision to return to fire suppression and rescue activities.

7. The present investigation developed a perceptual hyperthermia index in response to a need in fire suppression and rescue. The PHI may be beneficial in other populations such as in military personnel and football players. 
APPENDIX A

METABOLIC RATE CATEGORIES 


\section{A.1 EXAMPLES OF ACTIVITIES WITHIN METABOLIC RATE CATEGORIES}

\begin{tabular}{l|l}
\hline Categories & Example Activities \\
\hline Resting & $\begin{array}{l}\text { Sitting quietly } \\
\text { Sitting with moderate arm movement }\end{array}$ \\
\hline Light & $\begin{array}{l}\text { Sitting with moderate arm and leg movements } \\
\text { Standing with light work at machine or bench using mostly arms } \\
\text { Using a table saw } \\
\text { Standing with light or moderate work at machine or bench and some walking } \\
\text { about }\end{array}$ \\
\hline Moderate & $\begin{array}{l}\text { Scrubbing in a standing position } \\
\text { Walking about with moderate lifting or pushing } \\
\text { Walking on level at 6 Km/hr while carrying 3 kg weight load }\end{array}$ \\
\hline Heavy & $\begin{array}{l}\text { Carpenter sawing by hand } \\
\text { Shoveling dry sand } \\
\text { Heavy assembly work on a noncontinuous basis } \\
\text { Intermittent heavy lifting with pushing or pulling (e.g., pick-and-shovel work) }\end{array}$ \\
\hline Very Heavy & \begin{tabular}{l} 
Shoveling wet sand \\
\hline
\end{tabular}
\end{tabular}


APPENDIX B

STANDARDIZED INSTRUCTIONS FOR PERCEPTUAL MEASURES 


\section{B.1 INSTRUCTIONS FOR OMNI-RPE}

This scale contains numbers from 0 to 10 that will be used to rate the perception of physical exertion. The perception of physical exertion is defined as the intensity of the subjective effort, strain, discomfort and/or fatigue that you feel during an exercise task. We use this scale so that you may translate into numbers your feelings of exertion while exercising. These feelings should be general about the body as a whole.

The range of numbers on the scale should represent a range of feelings from "Extremely Easy" to "Extremely Hard". In order to help you select a number that corresponds to your subjective feelings consider the following. When the exercise feels between "Extremely Easy" and "Easy" respond with a number 1 . An example of when you would rate a number 1 would be when you encounter the same feelings as you have when you are walking very slowly.

When the exercise feels between "Hard" and "Extremely Hard" respond with a number 9. For example, a rating of 9 would be appropriate when your feelings of exertion are the same as your memory of how you felt during the most physically exhaustive work you have ever done.

When rating, think of your feelings associated with the numbers 1 and 9 first. Then, think of the exertion associated with the exercise at the moment and make your judgment. If the exertion feels less than a 1 or greater than a 9, respond with a 0 or 10 , respectively. You should only rate a number 0 when you are at rest such as sitting down or standing around.

In summary,

1. You will be asked to give a rating of perceived exertion every minute of the test.

2. Give each rating by selecting any number from 0 to 10 that corresponds to the perception of exertion for your total body.

3. Try to estimate the degree of exertion as accurately as possible.

4. Do not underestimate or overestimate the exertion, simply rate your feelings caused by the exercise at the moment.

5. There are no right or wrong answers.

6. Start with any number that is appropriate 


\section{B.2 INSTRUCTIONS FOR OMNI-THERMAL SENSATION}

This scale contains numbers from 1 to 5 that will be used to rate the perception of thermal sensation. The perception of thermal sensation is defined as the subjective feeling of heat. We use this scale so that you may translate into numbers how hot you feel. These feelings should be general about the body as a whole.

The range of numbers on the scale should represent a range of feelings from "Comfortable" to "Very Hot". In order to help you select a number that corresponds to your subjective feelings consider the following. When you feel "Comfortable", respond with a number 1. When you feel "Very Hot", respond with a number 5. 
APPENDIX C

OMNI SCALES 


\section{C.1 OMNI-RPE FOR WALK/RUN EXERCISE}

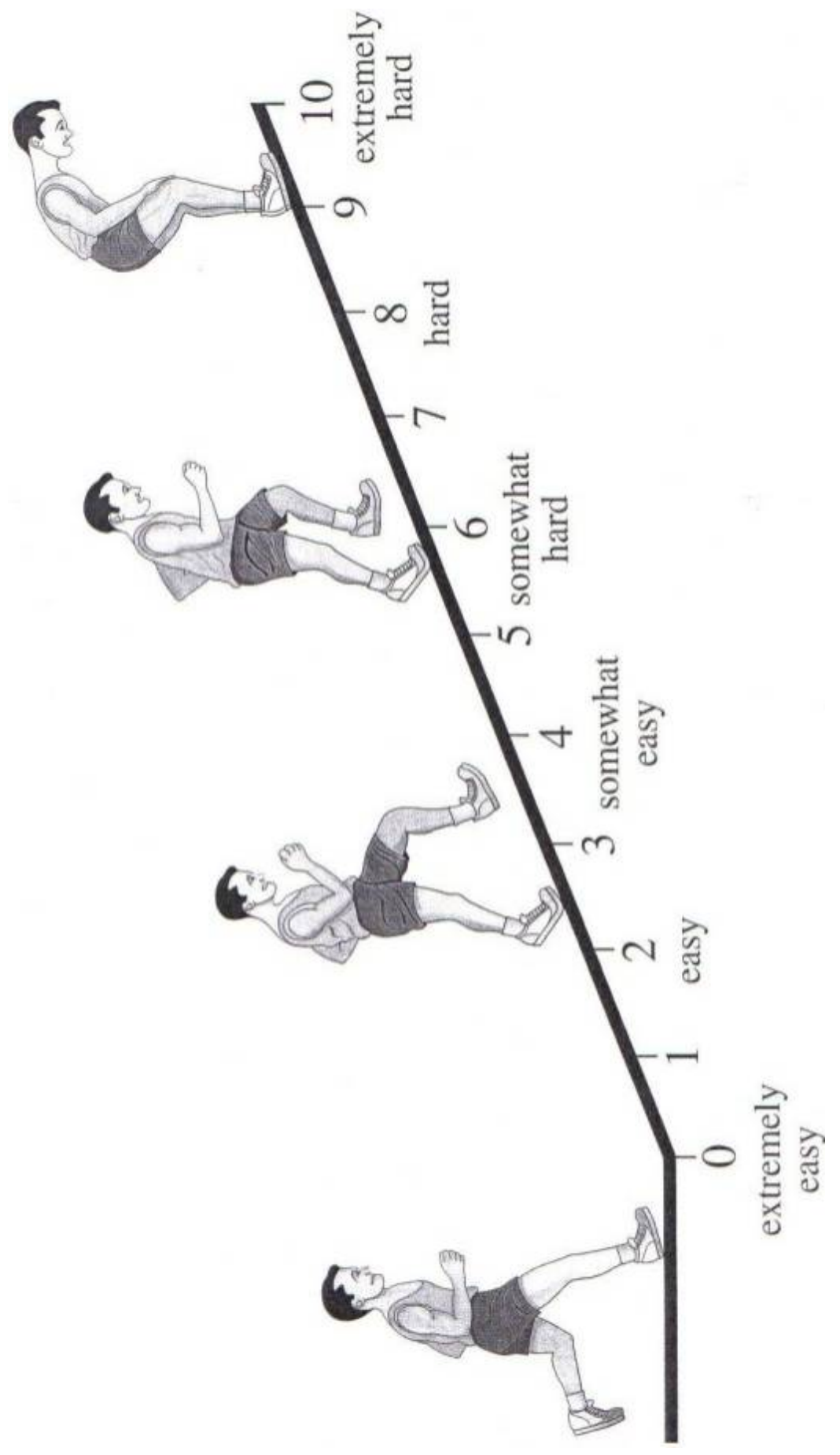




\section{C.2 OMNI-THERMAL SENSATION}

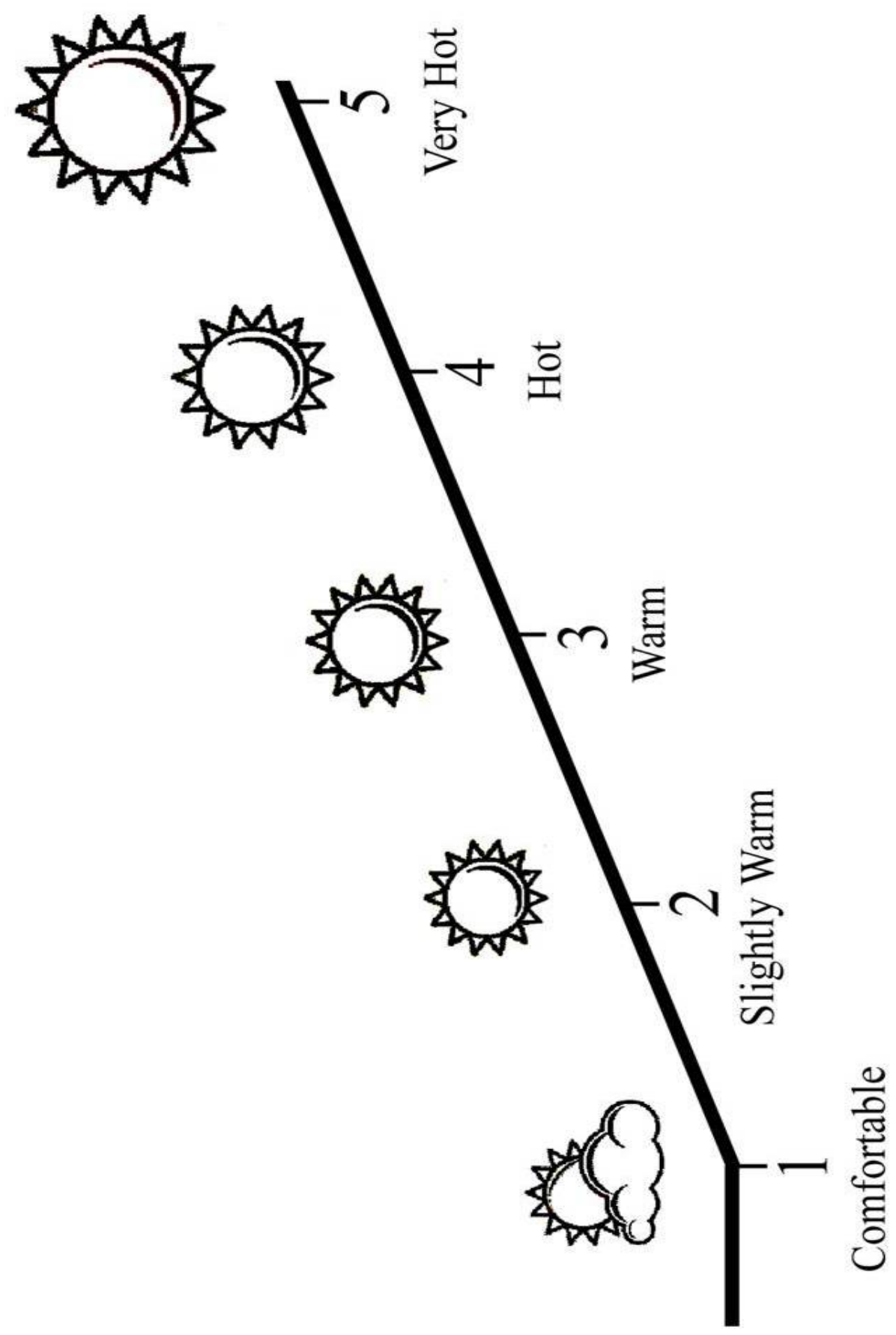




\section{BIBLIOGRAPHY}

1. ACGIH. (2001). Heat Stress and Strain. Cincinnati, OH: American Conference of Governmental Industrial Hygienists.

2. Belding, H. S., \& Hatch, T. F. (1955). Index for Evaluating Heat Stress in terms of Resulting Physiological Strain. Heat Pip Air Condit , 27, 129-36.

3. Bigland-Ritchie, B., Jones, D. A., \& Woods, J. J. (1978). Central and Peripheral Fatigue in Sustained Maximum Voluntary Contractions of Human Quadriceps Muscle. Clin Sci Mol Med , 54, 609-14.

4. Bilzon, J. L., Scarpello, E. G., Smith, C. V., Ravehill, N. A., \& Rayson, M. P. (2001). Characterization of the Metabolic Demands of Simulated Shipboard Royal Navy FireFighting Tasks. Ergonomics , 44, 766-80.

5. Binkley, H. M., Beckett, J., Casa, D. J., Kleiner, D. M., \& Plummer, P. E. (2002). National Athletic Trainers' Association Position Statement: Exertional Heat Illnesses. $J$ Athl Train , 37 (3), 329-43.

6. Bonauto, D., Anderson, R., Rauser, E., \& Burke, B. (2007). Occupational Heat Illness in Washington State, 1995-2005. Am J Ind Med , 50, 940-50.

7. Bouchama, A., \& Knochel, J. P. (2002). Heat Stroke. N Engl J Med , 346 (25), 1978-88.

8. Boulant, J. R. (1997). Thermoregulation. In P. A. Mackowiak (Ed.), Fever: Basic Mechanisms and Management (pp. 35-58). Philadelphia, PA: Lippincott-Raven.

9. Brake, R., \& Bates, G. (2002). A Valid Method for Comparing Rational and Empirical Heat Stress Indices. Ann Occup Hyg , 46 (2), 165-74.

10. Brearley, M. B., \& Finn, J. P. (2007). Responses of Motor-Sport Athletes to V8 Supercar Racing in Hot Conditions. International Journal of Sports Physiology and Performance , $2(2), 182-91$.

11. Carter III, R., Cheuvront, S. N., \& Sawka, M. N. (2006). Sports Science Exchange: Heat Related Illnesses. Barrington, IL: Gatorade Sports Science Institute. 
12. Casa, D. J., Becker, S. M., Ganio, M. S., Brown, C. M., Yeargin, S. W., Roti, M. W., et al. (2007). Validity of Devices that Assess Body Temperature during Outdoor Exercise in the Heat. J Athl Train , 42 (3), 333-42.

13. Castellani, J. W. (2003). Physiology of Heat Stress. In L. E. Armstrong (Ed.), Exertional Heat Illnesses (pp. 1-15). Champaign, IL: Human Kinetics.

14. Cheung, S. S. (2007). Neuropsychologial Determinants of Exercise Tolerance in the Heat. In H. S. Sharma (Ed.), Progress in Brain Research (Vol. 162, pp. 45-60). Elsevier B. V.

15. Cheung, S. S., \& McLellan, T. M. (1998). Influence of Hydration Status and Fluid Replacement on Heat Tolerance while wearing NBC Protective Clothing. Eur J Appl Physiol Occup Physiol , 7, 139-48.

16. Crewe, H., Tucker, R., \& Noakes, T. D. (2008). The Rate of Increase in Rating of Perceived Exertion Predicts the Duration of Exercise to Fatigue at a Fixed Power Output in Different Environmental Conditions. Eur J Appl Physiol , 103, 569-77.

17. Department of the Army and Air Force. (2003). Heat Stress Control and Heat Casualty Management. Retrieved from http://www.army.mil/usapa/med/DR_pubs/dr_a/pdf/tbmed507.pdf

18. Ducharme, M. B. (2006). Heat Stress of Helicopter Aircrew Wearing Immersion Suit. Industrial Health , 46, 433-40.

19. Epstein, Y., \& Moran, D. S. (2006). Thermal Comfort and the Heat Stress Indices. Industrial Health , 44, 388-98.

20. Gagge, A. P., Stolwijk, J. A., \& Hardy, J. D. (1967). Comfort and Thermal Sensations and Associated Physiological Responses at Various Ambient Temperatures. Environ Res $, 1,1-20$.

21. Galloway, S. D., \& Maughan, R. J. (1997). Effects of Ambient Temperature on the Capacity to Perform Prolonged Cycle Exercse in Man. Med Sci Sports Exerc , 29, 124049.

22. Gandevia, S. C. (2001). Spinal and Supraspinal Factors in Human Muscle Fatigue. Physiol Rev , 81 (4), 1725-89.

23. Gant, N., Atkinson, G., \& Williams, C. (2006). The Validity and Reliability of Intestinal Temperature during Intermittent Running. Med Sci Sports Exerc , 38 (11), 1926-31.

24. Gardner, J. W., Kark, J. A., Karnei, K., Sanborn, J. S., Gastaldo, E., Burr, P., et al. (1996). Risk Factors Predicting Exertional Heat Illness in Male Marine Corps Recruits. Med Sci Sports Exerc , 28 (8), 939-44. 
25. Greenes, D. S., \& Fleisher, G. R. (2004). When Body Temperature Changes, does Rectal Temperatures Lag? J Pediatr , 144, 824-26.

26. Greenes, D., \& Fleisher, G. R. (2001). Accuracy of a Noninvasive Temporal Artery Thermometer for use in Infants. Arch Pediatr Adolesc Med , 155, 376-81.

27. Haldane, J. S. (1905). The Influence of High Air Temperature. J Hyg , 5, 494-513.

28. Hocking, C., Lau, W. M., Silberstein, R., Roberts, W., \& Stough, C. (2000). The Effects of Thermal Strain on Cognition. Defense Science and Technology Organisation. Melbourne, Austraila: DSTO Aeronautical and Maritime Research Laboratory.

29. Holmer, I. (2006). Protective Clothing in Hot Environments. Industrial Health , 44, 40413.

30. Holmer, I., \& Gavhed, D. (2007). Classification of Metabolic and Respiratory Demands in Fire Fighting Activity with Extreme Workloads. Applied Ergonomics , 38, 45-52.

31. Hostler, D., Gallagher Jr, M., Goss, F. L., Seitz, J. R., Reis, S. E., Robertson, R. J., et al. (2009). The Effect of Hyperhydration on Physiological and Perceived Strain during Treadmill Exercise in Personal Protective Equipment. Eur J Appl Physiol , 105 (4), 60713.

32. Houghton, F. C., \& Yaglou, C. P. (1923). Determining Equal Comfort Lines. J Am Soc Heat Vent Engrs , 29, 165-76.

33. Jackson, A. S., \& Pollack, M. L. (1985). Practical Assessment of Body Composition. Physician Sportsmed , 13 (5), 76-90.

34. Kistemaker, J. A., Den Hartog, E. A., \& Daanen, H. A. (2006). Reliability of an Infrared Forehead Skin Thermometer for Core Temperature Measurements. J Med Eng Technol , 30, 252-61.

35. Kivimaki, M., \& Lusa, S. (1994). Stress and Ccognitive Performance of Fire Fighters during Smoke-Diving. Stress Med , 10, 63-8.

36. Kolka, M. A., Quigley, M. D., Blanchard, L. A., Toyota, D. A., \& Stephenson, L. A. (1993). Validation of a Temperature Telemetry System during Moderate and Strenuous Exercise. $J$ Therm Biol , 18, 203-10.

37. Latzka, W. A., Sawka, M. N., Montain, S. J., Skrinar, G. S., Fielding, R. A., Motatt, R. P., et al. (1998). Hyperhydration: Tolerance and Cardiovasular Effects during Uncompensable Exercise-Heat Stress. J Appl Physiol , 84 (6), 1858-64.

38. Lee, S. M., Williams, W. J., \& Fortney Schneider, S. M. (2000). Core Temperature Measurement during Supine Exercise: Esophageal, Rectal, and Instestinal Temperatures. Aviat Space Environ Med , 71, 939-45. 
39. Leon, L. R. (2006). The Thermoregulatory Consequences of Heat Stroke: Are Cytokines Involved? J Therm Biol , 31, 67-81.

40. Lind, A. R., \& Hellon, R. F. (1957). Assessment of Physiologic Severity of Hot Climate. $J$ Appl Physiol , 11, 35-40.

41. Low, D. A., Vu, A., Brown, M., Davis, S. L., Keller, D. M., Levine, B. D., et al. (2007). Temporal Thermometry Fails to Track Body Core Temperature during Heat Stress. Med Sci Sports Exerc , 39 (7), 1029-35.

42. Maughan, R. J., Shirreffs, S. M., \& Watson, P. (2007). Exercise, Heat, Hydration and the Brain. J Amer College Nutr , 26 (5), 604S-612S.

43. McLellan, T. M., \& Cheung, S. S. (2000). Impact of Fluid Replacement on Heat Storage while wearing Protective Clothing. Ergonomics , 43, 2020-30.

44. McLellan, T. M., \& Selkirk, G. A. (2006). The Management of Heat Stress for the Firefighter: A Review of Work Conducted on Behalf of the Toronto Fire Service. Industrial Health , 44, 414-26.

45. McPherson, M. J. (1992). The Generalisation of ACP. Proceedings 5th Internat Mine Vent Cong, (pp. 27-35). Johannesburg.

46. Moran, D. S. (2000). Stress Evaluation by the Physiological Strain Index (PSI). J Basic Clin Physiol Pharmacol , 11, 403-23.

47. Moran, D. S., \& Epstein, Y. (2006). Evaluation of the Envrionmental Stress Index (ESI) for Hot/Dry and Hot/Wet Climates. Industrial Health , 46, 399-403.

48. Moran, D. S., \& Mendal, L. (2002). Core Temperature Measurement: Methods and Current Insights. Sports Med , 32 (14), 879-85.

49. Moran, D. S., Pandolf, K. B., Shapiro, Y., Heled, Y., Shani, Y., Matthew, W. T., et al. (2001). An Environmental Stress Index (ESI) as a Substitute for the Wet-Bulb Globe Temperature (WBGT). J Thermal Biol , 26, 427-31.

50. Moran, D. S., Shitzer, A., \& Pandolf, K. B. (1998). A Physiological Strain Index to Evaluate Heat Stress. Am J Physiol. Regul Integr Comp Physiol , 275, R129-R134.

51. Morrison, S., Sleivert, G. G., \& Cheung, S. S. (2004). Passive Hyperthermia Reduces Voluntary Activation and Isometric Force Production. Eur J Appl Physiol, 91, 729-36.

52. Mower, G. D. (1976). Perceived Intensity of Peripheral Thermal Stimuli is Independent of Internal Body Temperature. J Comp Physiol Psychol , 90, 1152-55.

53. Nielsen, B., \& Nybo, L. (2003). Cerebral Changes during Exercise in the Heat. Sports Med , 33 (1), 1-11. 
54. Nielsen, B., Hyldig, T., Bidstrup, F., Gonzalez-Alonso, J., \& Christoffersen, G. R. (2001). Brain Activity and Fatigue during Prolonged Exercise in the Heat. Pflugers Arch Eur J Physiol , 442, 41-8.

55. NIOSH. (1986). Criteria for a Recommended Standard: Occupational Exposure to Hot Envrionments. DHHS (NIOSH) Publication 86-113 (pp. 101-114). Washington, D.C.: National Institute for Occupational Safety and Health.

56. Northington, W. E., Suyama, J., Goss, F. L., Randall, C., Gallagher, M., \& Hostler, D. (2007). Physiological Repsonses during Graded Treadmill Exercise in ChemicalResistant Personal Protective Equipment. Prehospital Emergency Care , 11, 394-8.

57. Nybo, L. (2007). Exercise and Heat Stress: Cerebral Challanges and Consequences. In H. S. Sharma (Ed.), Progress in Brain Research (Vol. 162, pp. 29-43). Elevier B. V.

58. Nybo, L., \& Nielsen, B. (2001). Hyperthermia and Central Fatigue during Prolonged Exericse in Humans. J Appl Physiol , 91, 1055-60.

59. Nybo, L., Moller, K., Volinaitis, S., Nielsen, B., \& Secher, N. H. (2002). Effects of Hyperthermia on Cerebral Blood Flow and Metabolism during Prolonged Exercise in Humans. J Appl Physiol , 93, 58-64.

60. O'Brien, C., Hoyt, R. W., Buller, M. J., Castellani, J. W., \& Young, A. J. (1998). Telemetry Pill Measurement of Core Temperature in Humans during Active Heating and Cooling. Med Sci Sports Exerc , 30, 468-72.

61. Parsons, K. (2006). Heat Stress Standard ISO 7243 and its Global Application. Industrial Health , 44, 368-79.

62. Petruzzello, S. J., Gapin, J. I., Snook, E., \& Smith, D. L. (2009). Perceptual and Physiological Heat Strain: Examination in Firefighters in Laboratory- and Field-based Studies. Ergonomics , 1-8.

63. Price, M. J., \& Campbell, I. G. (2002). Thermoregulatory Responses during Prolonged Upper-body Exercise in Cool and Warm Conditions. Journal of Sports Sciences , 20, 519-27.

64. Robertson, R. J. (2004). Percevied Exertion for Practitioners: Rating Effort with the OMNI Picture System. Champaign, IL: Human Kinetics.

65. Sawka, M. N., \& Wenger, C. B. (1998). Physiologic Responses to Actue Exercise Heat Stress. In K. B. Pandolf, M. N. Sawka, \& R. R. Gonzalez (Eds.), Human Performance Physiology and Environmental Medicine ni Terrestrial Extremes (pp. 97-151). Indianapolis, IN: Benchmark Press.

66. Smith, D. L., Manning, T. S., \& Petruzzello, S. J. (2001). Effect of Strenuous Live-Fire Drills on Cardiovascular and Psychological Responses of Recruit Firefighters. Ergonomics , 44 (3), 244-54. 
67. Stephenson, A., Quigley, M. D., Blanchard, L. A., Toyota, D. A., \& Kolka, M. A. (1992). Validation of Two Temperature Pill Telemetry Systems in Humans during Moderate and Strenuous Exercise. U.S. Army Research Institute of Environmental Medicine.

68. Tennebaum, J., Sohar, E., Adar, R., Gilat, T., \& Yaski, D. (1961). The Physiological Significance of the Cumulative Discomfort Index (Cum DI). Harefuah , 60, 315-9.

69. Thom, E. C. (1959). The Dicomfort Index. Weatherwise , 12, 57-60.

70. Tikuisis, P., McLellan, T. M., \& Selkirk, G. (2001). Perceptual versus Physiological Heat Strain during Exercise-Heat Stress. Med Sci Sports Exer , 34 (9), 1454-61.

71. Todd, G., Butler, J. E., Taylor, J. L., \& Gandevia, S. C. (2005). Hyperthermia: A Failure of the Motor Cortex and the Muscle. J Physiol , 563, 621-31.

72. Toner, M. M., Drolet, L. L., \& Pandolf, K. B. (1986). Perceptual and Physiological Responses during Exercise in Cool and Cold Water. Percept Motor Skills , 62 (1), 21120 .

73. Utter, A. C., Robertson, R. J., Green, J. M., Suminski, R. R., McAnulty, S. R., \& Nieman, D. C. (2004). Validation of the Adult OMNI Scale of Perceived Exertion for Walking/Running Exercise. Med Sci Sports Exerc , 36 (10), 1776-80.

74. Vernon, H. M., \& Warner, C. G. (1932). The Influence of the Humidity of the Air on Capacity for Work at High Temperatures. J Hyg , 32, 431-62.

75. von Heimburg, E. D., Rasmussen, A. K., \& Medbo, J. I. (2006). Physiological Responses of Firefighters and Performance Predictors during a Simulated Rescue of Hospital Patients. Ergonomics , 49 (2), 111-126.

76. Wallace, R. F., Kriebel, D., Punnett, L., Wegman, D. H., Wenger, C. B., Gardner, J. W., et al. (2006). Risk Factors for Recruit Exertional Heat Illness by Gender and Training Period. Aviat Space Envrion Med , 77 (4), 415-21.

77. Winslow, C. E., Herrington, L. P., \& Gagge, A. P. (1938). Physiological Reactions and Sensations of Pleasantness under varying Atmospheric Conditions. Trans ASVHE , 44, $179-96$.

78. Yaglou, C. P., \& Minard, D. (1957). Control of Heat Casualties at Military Training Centers. Am Med Ass Arch Ind Hlth , 16, 302-16. 